\title{
Les Territoires de l'eau et la décentralisation. La gouvernance de bassin versant ou les limites d'une évidence
}

\section{Stéphane Ghiotti}

\section{(2) OpenEdition}

1 Journals

Édition électronique

URL : http://journals.openedition.org/developpementdurable/1742

DOI : 10.4000/developpementdurable.1742

ISSN : 1772-9971

Éditeur

Association DD\&T

Référence électronique

Stéphane Ghiotti, «Les Territoires de l'eau et la décentralisation. La gouvernance de bassin versant ou les limites d'une évidence », Développement durable et territoires [En ligne], Dossier 6 | 2006, mis en ligne le 10 février 2006, consulté le 19 avril 2019. URL : http://journals.openedition.org/ developpementdurable/1742 ; DOI : 10.4000/developpementdurable.1742

Ce document a été généré automatiquement le 19 avril 2019

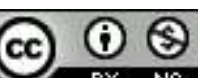

Développement Durable et Territoires est mis à disposition selon les termes de la licence Creative Commons Attribution - Pas d'Utilisation Commerciale 4.0 International. 


\title{
Les Territoires de l'eau et la décentralisation. La gouvernance de bassin versant ou les limites d'une évidence
}

\author{
Stéphane Ghiotti
}

1 La mise en œuvre conjointe en France de la directive cadre européenne (DCE) ${ }^{1}$, de « l'acte II » de la Décentralisation et de la Charte de l'environnement renforce considérablement une tendance déjà ancienne à la territorialisation de la politique de l'eau. Le territoire acquiert un nouveau statut, celui de concept opératoire aussi bien dans le domaine de l'aménagement du territoire que dans celui de la gestion de l'eau. Aujourd'hui, gage de l'adaptation de ces politiques aux contextes et aux enjeux locaux et cherchant la mise en cohérence de l'action publique, la territorialisation est sensée par cette proximité favoriser la démocratie locale à cette échelle et assurer l'efficacité sociale et économique des réformes. En France, c'est le concept de bassin versant qui sert de cadre à la gestion territoriale de l'eau. Mais si cette approche fonctionnaliste traditionnelle par bassin est malgré tout renforcée par le vaste mouvement de recomposition organisationnelle et politique en cours, ce dernier fait émerger d'autres constructions dont les logiques ne sont pas toujours en adéquation avec le "modèle ». A côté DU territoire de l'eau (ré ?) émergent ou se constituent DES territoires de l'eau, pas toujours en lien avec les approches naturelles ou environnementales mais plus en phase avec les logiques intercommunales, départementales et régionales issues de la décentralisation.

2 Les rapports tissés entre décentralisation et bassin versant sont étroits, riches d'indications et se structurent vraiment à partir du XIX ${ }^{\text {ème }}$ siècle. L'effort de conceptualisation autour du bassin versant couplé à la mise en perspective géographique et historique nous renseignent sur les arrière-fonds sociétaux dans lesquels émergent ces réformes et les enjeux politiques, territoriaux et institutionnels qui les accompagnent. $\mathrm{Si}$ nous nous interrogerons sur les processus et les enjeux inhérents à cette nouvelle étape 
de la territorialisation de la politique de l'eau dans ses fonctions et ses formes, une mise en perspective spatiale et temporelle de ce processus est un préalable nécessaire. Comment la dimension territoriale s'est-elle progressivement construite, développée et imposée dans les politiques de gestion de l'eau? Comment ces dernières, sous l'impulsion des lois de décentralisation, se sont-elles progressivement intégrées dans les problématiques plus globales du développement territorial et avec quelles conséquences? Cet article interrogera d'abord l'apparente évidence des approches territoriales et par bassin versant pour ensuite les replacer dans les débats et les enjeux liés à l'organisation et à l'administration du territoire depuis les années 1980.

3 Le processus de territorialisation est défini par Angelo Turco (1997, p. 233) comme " l'ensemble des procédures à travers lesquelles un territoire se forme et évolue ». Les nombreux travaux sur le concept de territoire (Frémont, 1976 ; Raffestin, 1980, 1986 ; Barel, 1981 ; Debarbieux, 1988 ; Gumuchian, 1991 ; Di Méo, 1998) permettent d'affiner la définition de la territorialisation en replaçant ce concept au sein des interrelations entre l'homme et son environnement. En essayant de proposer une synthèse des différentes approches en géographie sociale on peut dire que la territorialisation se caractérise par la mise en place d'une organisation et d'une structuration nouvelles et spécifiques, matérielles et/ou idéelles, d'une portion d'espace par un groupe social. Nomination, délimitation, patrimonialisation (Di Méo, 1994) font également partie de ce processus en constante évolution.

4 Concernant les politiques de l'eau, le processus de territorialisation a également été abordé. De nombreuses approches se sont en effet largement attachées à démontrer le rôle central de l'eau dans l'organisation et l'appropriation de l'espace, dans la construction des représentations collectives et individuelles ou encore dans la structuration des relations sociales et économiques des sociétés locales (Marié, 1982, 1983 ; Drain, 1998, Béthemont, 2000). De récents travaux (Béthemont, Faggi, Zoungrana Tanga, 2003) proposent dans une optique de formalisation une analyse fine d'un processus de territorialisation hydraulique et placent ce dernier à l'articulation de trois polarités: les objectifs de la politique poursuivie (entre hydraulique productive et hydraulique stratégique), les stratégies ou intensité du processus (entre procédures fortes et procédures simples) et la qualité des ressources employées (entre matérialisation du territoire - infrastructures - et contrôle du territoire - processus gestionnaires, nouvelles normes, etc.). Ce rapide panorama théorique et conceptuel nous permettra donc d'analyser la recomposition des relations entre acteurs, ressources et territoires à travers un fil conducteur que sera la progressive territorialisation par bassin versant. Il ne s'agit plus d'aborder l'approche territoriale seulement en terme de cadre d'analyse mais de la considérer également comme un objet d'analyse en tant que tel (Ghiotti, 2001). Un retour sur les temps et les formes de la conceptualisation du bassin versant servira de grille de lecture. Trois facteurs majeurs se dégagent: l'évolution des paradigmes sur la connaissance du cycle de l'eau, le rôle de l'Etat dans sa volonté de contrôle de cette ressource, l'évolution des rapports société/eau/territoire.

5 Le travail rétrospectif visant à éclairer les différents processus de territorialisation de la gestion de l'eau veut élargir la problématique et proposer sa re-formulation dans un cadre historique et géographique plus large. Il s'appuie sur l'observation et l'analyse des rapports entre les représentations, les usages de la ressource par les différents utilisateurs et les contextes socio-économiques, démographiques et techniques. Les changements de représentation et d'usages influencent profondément les modalités de 
gestion qui leur sont liés. Cette démarche vient en complément de celle développée plus récemment qui tend à relativiser fortement la place, aujourd'hui centrale, de la gestion des flux dans la gestion de l'eau (Narcy, Mermet, 2003).

6 La gestion territoriale de l'eau est une forme d'organisation ancienne, évolutive (Marié, Larcena, Dérioz, (dir.), 1999) et qui s'est développée sur l'ensemble du territoire national (Billaud, 1984; Picon, 1978; Honegger, 1990, Manceron, 2003). Elle se manifeste sous des formes multiples, des échelles et des découpages spatiaux variés et intervient dans les processus de gestion avec plus ou moins d'intensité selon les espaces. Par exemple, la gestion territoriale est une forme très développée d'organisation sociale déjà en place à la Révolution, notamment dans les montagnes méditerranéennes où domine une gestion villageoise à l'échelle du versant. Dans les montagnes méditerranéennes par exemple, la gestion territoriale est une forme très développée d'organisation sociale, déjà en place à la Révolution, où domine une gestion communautaire des ressources et où l'échelle privilégiée est celle du versant. Ainsi, ces espaces nous donnent une telle représentativité du phénomène qu'il est difficile de les contourner en terme d'analyse. La lecture détaillée des archives montre en effet très clairement que ce phénomène s'individualise par une très forte spécificité, au vu des usages de la ressource parmi lesquels dominent très largement l'irrigation, le curage et l'endiguement (Haghe, 1998, p. 375).

7 La lutte contre l'inégalité spatiale et temporelle de la ressource, la mise en place de son partage participent pleinement à l'organisation sociale et territoriale des différentes communautés en France (Aubriot, Jolly, (coord.) 2002) mais également à l'étranger comme au Portugal par exemple (Wateau, 2002a). L'accès à l'eau, son partage par l'identification des droits d'eau s'effectuent à travers des pratiques sociales multiples (les corvées comme l'entretien et le nettoyage des canaux, des prises d'eau, organisation des tours d'eau, etc.) et s'opèrent à l'aide de procédés de types informel (tradition orale), matériel (canne de roseau (Wateau, 2001), pierre de partage de l'eau (Wateau, 2002b) voire sonore (Wateau, 2004). Les multiples liens que tissent l'eau et les territoires ne s'établissent donc pas selon un processus linéaire dans le temps et dans l'espace et selon des modalités et des découpages uniques. La gestion territoriale de l'eau connaît trois grandes périodes où s'impose une conception dominante quant à l'utilisation de la ressource (Cf. schéma $\left.{ }^{\circ} 1\right)$.

8 L'eau, des XVIII ${ }^{\text {ème }}$ et XIX ${ }^{\text {ème }}$ siècles présente une forme d'organisation très territorialisée (schéma 1, période a). Elle se caractérise d'abord comme une source de solidarités informelles entre les différentes communautés d'usagers organisées localement par l'aménagement des versants (Ghiotti, 2000), (terrasses et réseaux d'irrigation par béalières) ou des plaines d'inondation (colmatage et construction d'épis) par exemple. Cette volonté de maîtrise de l'environnement physique est plutôt de nature agressive en montagne comme en plaine, ce qui tend à relativiser une solidarité « naturelle » de bassin entre l'amont et l'aval. L'ampleur des transformations durant tout le XIX ${ }^{\text {ème }}$ fut-elle, « un remodelage total des versants, créant une topographie entièrement nouvelle, transformant profondément les écoulements des eaux» (Frapa, 1999, p. 113) que les communautés villageoises de plaine ont à leur tour, dans le même contexte démographique et socioéconomique, développé des pratiques de gestion agressive pour assurer leur protection et leur survie alimentaire. Emmanuelle Gautier (1996) note ainsi que la politique d'endiguement sur le Buëch (05) entre 1830 et 1860 est particulièrement agressive au point de modifier durablement la dynamique fluviale. L'eau est destinée à des usages locaux essentiellement domestiques, agricoles et artisanaux dont la répartition entre 
usagers, également locaux, s'effectue selon des coutumes et des codes propres à chaque communauté ce qui n'empêche pas un recours au droit et à la justice en période de crise. L'échelle de gestion privilégiée est celle - très territorialisée - de la communauté ou/et de son réseau d'irrigation quand les deux se confondent (Ruf, 2000).

Le XVIII ${ }^{\text {ème }}$ siècle et la première moitié du XIX ${ }^{\text {ème }}$ ont consacré l'eau comme un outil de production agricole au service de l'économie locale. Avec l'apparition de la Houille Blanche en 1869 et les débuts de la $2^{\text {nde }}$ révolution industrielle, les usages de l'eau enregistrèrent des modifications profondes d'affectation. Ainsi, des années 1870 jusqu'en 1960 (schéma 1, période b), la gestion territoriale de l'eau prend une nouvelle forme avec l'avènement de l'industrie et de sa logique productive. L'augmentation des besoins et la diversification des usages favorisent une appropriation de la ressource de plus en plus institutionnalisée entre usages, à des échelles d'aménagement supérieures et multiples par rapport à la période précédente. L'accès à l'eau déplace les conflits qui désormais ne s'effectuent plus tellement entre usagers mais plutôt entre usages (agricole, industriel, alimentation en eau potable). Les demandes en eau urbaine et industrielle sont devenues telles qu'elles nécessitent l'aménagement d'infrastructures capables de mobiliser la ressource de plus en plus loin des centres de consommation afin de régulariser l'approvisionnement. De telles pratiques d'aménagement cristallisent les oppositions autour de conflits territoriaux (amont - aval) comme le montre très bien Luc Berger pour l'Est du Massif central (Berger, 1998). La "solidarité » hydraulique entre l'amont - lieu de localisation des ressources mais également de prélèvement - et l'aval, lieu de consommation, se disloque complètement sous le double effet de la concurrence économique et des progrès technologiques qui éloignent de plus en plus socialement et spatialement les deux espaces. Deux types de territorialisation coexistent. Celle qui domine à cette époque repose sur une définition technique et un découpage hydraulique du territoire support des politiques d'aménagement et de gestion reposant sur des logiques très largement a-spatiales. Les relations entre la norme (celle de l'aménagement) et le territoire (celui des sociétés locales) n'est pas toujours aussi « rigide » et il arrive que la norme se plie, se déforme au contact de ces sociétés pour intégrer ce que Michel Marié (1983) appelle "l'épaisseur du territoire ». La poursuite des aménagements des secteurs amonts, la modernisation de l'agriculture et l'exode rural auront pour principales conséquences, notamment en montagne, d'inverser les tendances dans le système de domination de la période précédente, généralement au profit de la plaine. Le rôle de l'Etat s'accroît, en parallèle à un fractionnement des utilisations et une entrée progressive de l'eau dans la sphère marchande où elle acquiert un prix que les utilisateurs doivent payer pour accéder à la ressource. La valeur d'échange prend le pas sur la valeur d'usage.

Enfin, à partir des années 1960-1970 (schéma 1, période c), la longue tradition d'aménagement et d'utilisation agressive des rivières provoque des modifications de fonctionnement du système fluvial dont les conséquences mettent en péril la pérennité même des usages. Avec les décennies 1970 et 1980, la reconnaissance de la dimension " eau milieu » à côté de celle relative à « l'eau ressource » s'impose dans les politiques de gestion de l'eau. Elle ne fera que se renforcer du fait des progrès des connaissances scientifiques (concept d'hydrosystème), des revendications sociales autour de la qualité de la vie ainsi que des logiques politiques à l'œuvre (structuration du courant écologiste). L'heure est à la co-évolution des impératifs économiques et environnementaux. L'eau se trouve de plus en plus réappropriée socialement et acquiert le statut d'un patrimoine qu'il faut protéger et transmettre. Le bassin versant, avec ces nouvelles dimensions 
sociale, territoriale et environnementale supplante - au moins dans les discours - le bassin hydrologique et s'impose alors comme le territoire de la gestion de l'eau avec une reconnaissance institutionnelle et législative. D'abord à l'échelle nationale avec la loi sur l'eau de $1964^{2}$, puis à l'échelle locale avec celle de $1992^{3}$, instaurant le Schéma d'Aménagement et de Gestion des Eaux (SAGE) enfin à l'échelle européenne en 2000 avec la Directive Cadre Européenne (DCE) et les districts hydrographiques ${ }^{4}$.

L'approche des différentes organisations territoriales constituées autour de l'eau et de son exploitation illustre que la notion de ressource se structure autour de trois polarités majeures : économique, territoriale et environnementale. Suivant les époques, l'une ou l'autre des polarités s'est positionnée en élément dominant dans un système de régulation, sans pour autant en exclure les deux autres. C'est pourquoi les trois périodes identifiées ne renvoient pas à des systèmes indépendants et statiques, mais se conçoivent plutôt comme des organisations mouvantes en interrelation, où se recomposent les rapports entre « sphère publique », «sphère privée » et « sphère communautaire ».

Schéma $n^{\circ} 1$ : tendances et évolutions d'une gestion territoriale de l'eau (XVIIIeme $-X^{\text {eme }}$ siècle). Entre économie, territoire et environnement.

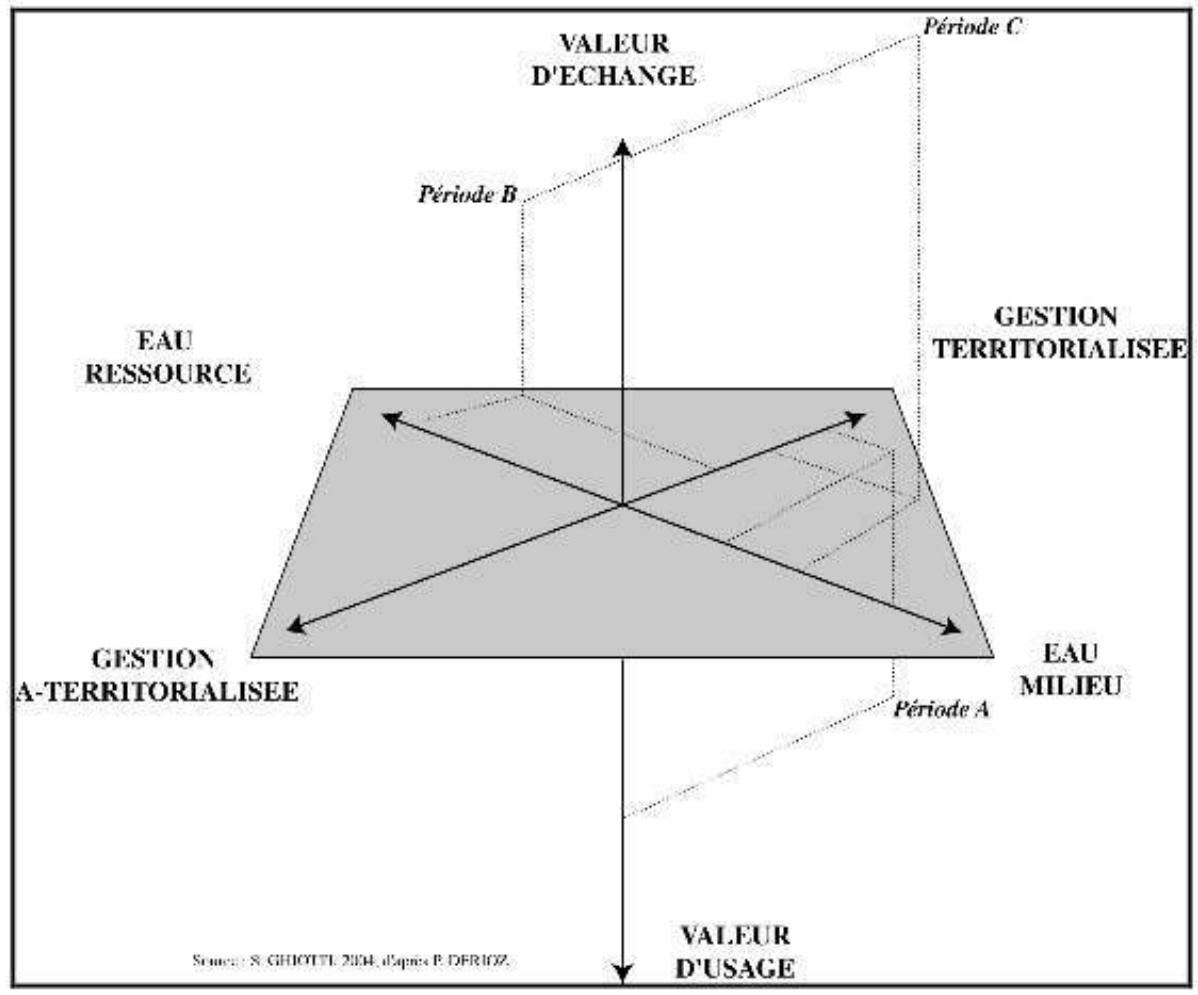

Retour texte période A, Retour texte période B, Retour texte période $\mathrm{C}$

12 [Agrandir le graphiquesch]

13 La territorialisation de la gestion de l'eau trouve ses racines bien avant la Révolution et évolue dans ses objectifs et ses échelles d'intervention. Si elle ne se confond réellement avec une approche par bassin versant que depuis une vingtaine d'années, les liens se tissent dans la seconde moitié du XIX ${ }^{\text {ème }}$ et l'essor de l'industrie où l'eau intègre la sphère productive et marchande. L'eau a certes toujours fait partie intégrante de l'activité économique des sociétés locales ; mais l'introduction de la logique marchande contribue, par l'augmentation des besoins et la diversification des usages, à redéfinir les 
problématiques dont le changement d'échelle lié à son exploitation et à sa distribution est l'élément central. L'eau cesse d'être une ressource locale où domine l'usage agricole. L'intégration de l'eau dans l'aménagement régional et national s'illustre par la variété et la puissance des enjeux complétées par celles des techniques et les appareils mobilisés pour y répondre.

Bien qu'il s'agisse d'un objet géographique présenté comme naturel, le bassin versant n'en demeure pas moins une forme de découpage de la nature qui renvoie toujours à une vision particulière du territoire et de sa finalité. Puisqu'il s'apparente à l'exercice du pouvoir et à sa délimitation, le découpage du territoire, aussi naturel soit-il, n'est pas neutre et s'apparente à un acte d'objectivation de celui-ci (Vanier, 1997). Un retour sur les différentes périodes de décentralisation permet d'effectuer ce décryptage conceptuel. En effet, c'est à l'occasion de ces débats que le bassin versant se trouve confronté avec d'autres territoires de l'eau, le plus souvent administratifs et notamment les départements. Les politiques relatives à l'organisation et à l'administration du territoire recherchent toujours les «bonnes limites» afin de substituer les découpages « immuables » aux découpages "relatifs et corrompus». La réduction progressive de la gestion territoriale de l'eau à la gestion par bassin versant s'effectue en deux temps, où à sa progressive formalisation succèdent la maturité puis l'opérationnalité. Ces différentes étapes témoignent des conflits, des ajustements multiples et souvent difficiles à établir entre "sphère publique ", "sphère privée » et "sphère communautaire ». Elles éclairent sous un angle nouveau la normalité de ce découpage naturel et les enjeux liés à la territorialisation de la politique de l'eau.

C'est Philippe Buache (1700-1773) qui le premier en 1752, a véritablement formalisé ce concept au sein d'une théorie fournissant un système interprétatif général à la répartition du relief terrestre en liant réseau hydrographique et orographie (Broc, 1991; Lagarde, 1987; Lagarde, 1998). Dans ce système, basé sur les divisions naturelles, les montagnes forment une ossature continue à l'échelle du globe, délimitant les bassins fluviaux et maritimes. Si ces considérations trouvent leur intérêt dans les domaines scientifiques et sociaux, elles trouvent leur prolongement dans le champ du politique. [Voir la carte de Ph. Buache]

$16 \mathrm{Au}$ cours des décennies 1750-1760, instrument de connaissance scientifique et clé de lecture interprétative du monde, le bassin hydrographique donne, par ses limites intangibles, les moyens à la monarchie, d'inscrire son autorité et de bâtir son administration. Par cette dimension scientifique absolue, il est devenu sous l'influence physiocratique, l'instrument de la rationalité économique et l'outil de la valorisation « des eaux utiles » dont la finalité productive opère la fonctionnalisation du milieu. Cette opération de vivification économique s'étend à la sphère sociale avant d'atteindre une dimension territoriale. Le bassin devient le cadre de la rationalisation des usages communautaires et, dès lors, de leur substitution au profit d'un territoire hydraulique homogène voué au marché comme en témoigne la carte du bassin de la Seine réalisé par Philippe Buache en $1766^{5}$. La rationalisation hydraulique se veut une uniformisation du territoire et place progressivement l'exploitation des eaux courantes dans la sphère marchande.

17 Avec Philippe Buache, les limites naturelles font leur apparition dans la désignation et la délimitation des objets géographiques. Ainsi, elles jettent aussi les bases de deux siècles de débats sur la pertinence et la concordance des découpages suivant qu'ils se réfèrent à l'ordre naturel ou à l'ordre politique, économique ou historique : « Cette façon de considérer 
notre globe ouvre une nouvelle carrière à la géographie. Il est peut-être plus intéressant de connaître la direction de ces chaînes de montagnes qui fournissent et dirigent les eaux du fleuve [... ] que de connaître les anciennes bornes d'un pays » (Buache, cité par Lagarde, 1998, p. 153). Les premières formes tangibles de ces oppositions émergèrent à la Révolution.

Les idées de Philippe Buache se retrouvent jusqu'à la Révolution où le concept de bassin hydrographique s'ancre véritablement dans une dimension politique. Elles émergent plus particulièrement au sein de deux débats, la réorganisation institutionnelle, réglementaire et politique des eaux courantes et le re-découpage du territoire, deux questions liées à la mise en œuvre de la départementalisation du territoire national (Ozouf-Marignier, 1989). Les motivations de l'Etat renvoient à des stratégies qui marquent sa volonté de réorganiser véritablement la vie politique, sociale et économique du pays. Dans la continuité du XVIII ${ }^{\text {ème }}$, l'eau est la pièce maîtresse du développement économique et les idées physiocratiques relancées par l'approche hygiéniste concourent toujours à l'augmentation et la rationalisation de la production agricole ainsi qu'aux échanges. Dans ce contexte et face à ces enjeux, l'action de l'Etat vise à s'imposer comme le seul acteur politique, à s'approprier de manière exclusive cette ressource et enfin à s'approprier le territoire en vue de son uniformisation. Pour ce faire, elle s'appuie sur un triptyque centralisateur, l'action législative et réglementaire par laquelle l'administration centrale garde l'autorité sur les cours d'eau navigables et flottables et transfère le reste à l'administration départementale, sur l'expertise assurée par les ingénieurs des Ponts et Chaussées et sur un territoire, le département afin de dépasser l'échelle communale jugée inopérante et trop soumise aux particularismes locaux.

Dans les discussions concernant le remplacement des anciennes Provinces par les départements, des interrogations se posent autour du respect ou non des limites naturelles pour les futurs découpages. S'il est effectif que les débats sur la départementalisation font référence au bassin hydrographique, les diverses revendications qui les motivent sont loin de revêtir un caractère absolu (Ozouf-Marignier, 2002). Tantôt revendiqué comme le meilleur découpage tantôt rejeté, le bassin hydrographique reflète ainsi assez fidèlement les hésitations des constituants et des sociétés locales sur l'attitude à tenir pour le nouveau découpage du territoire. Les référents naturels sont utilisés à la fois par les révolutionnaires soucieux d'imposer leur division du territoire et par les conservateurs partisans du statu quo territorial mais aussi économique et social. Le bassin versant devient un argument relatif, d'opportunité, utilisé par chacun des camps, opposant réforme et conservatisme, unité et ordre ancien, identité et particularisme contre rationalisation. Du point de vue territorial, il est mobilisé à la fois comme un facteur d'appropriation identitaire (homogénéité du territoire) mais aussi comme facteur de solidarité territoriale (complémentarité du territoire).

21 De cette période, le bassin garde cette expression de valeur refuge, d'opposition au centralisme tout en étant reconnu pertinent comme cadre administratif pour la gestion des ressources alors que scientifiquement sa validité est de plus en plus fortement remise en cause notamment par les géologues. Le bassin hydrographique quitte le seul registre des eaux courantes pour s'installer dans celui du découpage administratif et politique.

Après l'époque de sa structuration au plan territorial et scientifique, le bassin hydrographique connaît avec le XIX ${ }^{\text {ème }}$ siècle, une période où l'emprise réglementaire se développe dans ces deux domaines. La dimension territoriale recoupe les revendications décentralisatrices et régionalistes qu'accompagne également le capitalisme naissant. Il est l'expression de l'opposition au centralisme parisien, qu'il soit de nature politique, 
administrative ou encore scientifique. Ce retour en "grâce » de l'approche par bassin est particulièrement visible sous le Second Empire où les conditions politiques, économiques et scientifiques évoluent fortement. Le bassin hydrographique se trouve à la conjonction d'intérêts entre acteurs multiples, économiques et sociaux (industriels et aristocratie foncière de province), scientifiques (enjeux autour du contrôle de l'expertise) dans un contexte idéologique marqué par le retour d'un certain conservatisme. En réaction à la centralisation napoléonienne, le bassin hydrographique connaît alors son apogée à partir du milieu du XIX ${ }^{\text {ème }}$ siècle.

Schéma $\mathrm{n}^{\circ} 2$ : Le bassin versant, une alternative à la centralisation ?

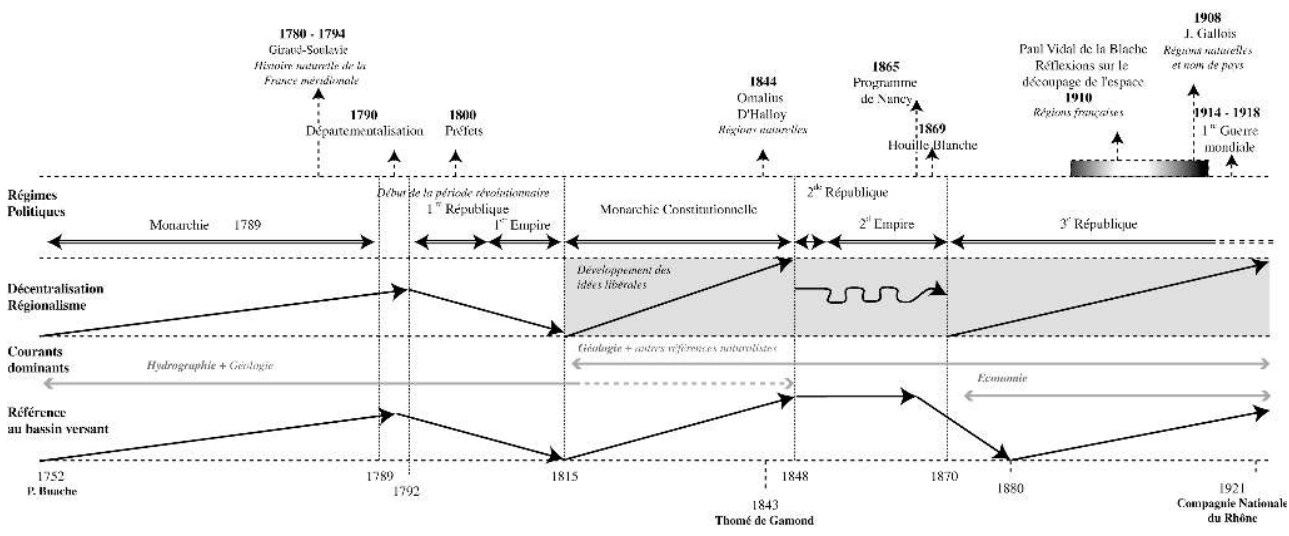

Source : Ghiotti.(S.), 2001.

selon les principaux usages, agricole, industriel et sanitaire. Cette multiplication des usages conjuguée à l'augmentation des besoins dans le cadre d'une gestion cloisonnée ne manque pas d'engendrer les premiers conflits. Face aux enjeux politiques et économiques liés à ce début de crise, la nécessité de mettre en œuvre une expertise globale embrassant l'ensemble des problématiques devient incontournable. Cette expertise doit se mettre en place au sein d'un territoire permettant non seulement cette globalisation des connaissances mais également l'optimisation économique de la ressource et de son utilisation. Bien que la tendance générale soit à la spécialisation et à l'éclatement des savoirs, les enjeux économiques poussent à la constitution d'une connaissance globale des eaux courantes. L'échelle du bassin devient donc le référentiel de réflexion et d'action. Cette nouvelle étape propulse l'approche par bassin au-delà des « pertinences » technicoscientifiques et administratives. Le bassin hydrographique intègre le champ de l'aménagement et du développement économique du territoire gage d'une véritable opérationnalité. L'exemple le plus significatif est donné par Thomé de Gamond en 1843 dont les propositions à l'échelle des cinq grands bassins hydrographiques, préfigurent très largement les Agences de bassin qui verront le jour un siècle plus tard (Haghe, 1998, p. 198 ; Bonnaud, 2000 ; Thomé de Gamond, 1871).

Cette nouvelle étape relance les querelles administratives et institutionnelles pour le contrôle et l'exercice de l'expertise entre agronomes et polytechniciens. Le mise en place d'une gestion par bassin correspond pour les premiers à une stratégie visant à casser le monopole de l'expertise des seconds et d'accéder par cette nouvelle territorialisation à un certain pouvoir par la reconnaissance scientifique et sociale de leurs compétences. 
26 La lutte contre un certain centralisme s'organise donc à cette époque autour des enjeux économiques, de l'expertise et du territoire de gestion non seulement à l'échelle nationale mais également à l'échelle locale comme en témoigne l'exemple des Associations Syndicales Hydrauliques (ASH). Ces associations, créées à la Révolution française, sont sensées encadrer les forces communautaires qui conservent un rôle actif dans l'usage des eaux courantes. Elles permettent de regrouper, hors du cadre communal, l'ensemble des communautés d'usagers d'un bassin hydrographique. L'idée est séduisante dans la mesure où ces associations donnent la possibilité aux solidarités riveraines et de bassin de s'exercer pleinement, sans une tutelle trop forte de l'administration centrale. Ces objectifs originels sont rapidement abandonnés par le noyautage des ces regroupements d'usagers par :

- l'aristocratie foncière, soucieuse de rentabiliser au maximum ses acquisitions en transférant les frais d'entretien sur les différentes communautés,

- l'Etat luttant contre les forces centrifuges et notamment les communautés, susceptibles de revendiquer une part d'autonomie et de pouvoir. Ce contrôle s'exerce par le biais des ingénieurs de Ponts et Chaussées qui gardent ainsi également la mainmise sur l'expertise.

Les réactions face au développement de cette nouvelle approche furent globalement marquées par le conservatisme qui légitime le rejet et l'opportunisme. A l'origine, les agronomes de la société des agriculteurs de France font du bassin versant, une circonscription capable de dépasser les antagonismes administratifs, surtout quand les cours d'eau sont séparés des départements. Ils voient, par la création d'un service hydraulique national, le moyen institutionnel de récupérer une partie du monopole de l'expertise. La conquête du pouvoir des agronomes face aux ingénieurs des Ponts échoue pour deux raisons : le poids du département et celui du monopole de l'expertise. Jean-P aul Haghe (1998, p. 583) indique «que si les limites territoriales des associations échappent au cadre territorial communal, elles gardent pourtant l'assise départementale ». On comprend aisément la réaction "des défenseurs " du département qui voient se développer un nouveau service qui risque fortement de les concurrencer tant du point de vue administratif que territorial. Le relatif succès des ASH s'apparente plus à une volonté de contrôle institutionnel des eaux courantes par l'administration en place qu'à une réelle envie de développer une nouvelle approche de la gestion à l'échelle locale.

Ces différents exemples montrent combien les logiques décentralisatrices participent à l'émergence et à la construction de territoires de l'eau multiples (ASH, département, bassin hydrographique) et les enjeux liés à « l'articulation » de ces territorialisations. Les interrelations entre technique, politique, économie et aménagement du territoire font émerger un autre découpage. Il repose, à l'instar des propositions de Thomé de Gamond, sur les cinq bassins hydrographiques se plaçant ainsi résolument à l'échelle régionale. Cette conjonction entre « expertise savante et projet politique » (Ozouf-Marignier, 2002, p. 73) est due à l'arrivée de nouveaux acteurs, les milieux économiques qui trouvent, via le lobbying des Chambres Consulaires, le moyen de mettre en place un régionalisme économique plus apte à servir leurs intérêts. Plusieurs propositions se succéderont: projet Béchard en 1851, Programme de Nancy en 1865, Régions Clémentel de 1917 et enfin les régions hydrauliques. Espaces où l'influence du fleuve, dans son utilisation, marque l'organisation et la structuration du territoire, elles sont issues de l'avènement de la Houille blanche et du développement industriel et démographique des pôles urbains. Elles serviront quelques années plus tard de modèle à la Compagnie Nationale du Rhône (CNR). Les enjeux politiques et économiques ${ }^{6}$ issus de la maîtrise de la force hydraulique, de sa 
gestion dans le cadre d'une problématique nationale d'aménagement et de développement du territoire posent des problèmes de réorganisation de celui-ci. La loi sur l'hydroélectricité de $1919^{7}$ entraîne un véritable bouleversement dans l'utilisation des eaux courantes. Si la rupture est d'abord juridique, les conséquences sont beaucoup plus générales et profondes et touchent l'organisation des eaux courantes dans son ensemble :

- l'action législative renforce le pouvoir de l'Etat. Elle participe à la création d'un nouveau bien, l'énergie motrice, qu'elle range dans le domaine privé de l'Etat

- la place centrale prise par l'Etat dans cette nouvelle organisation participe à son profit à un vaste mouvement d'homogénéisation :

- économique, avec la consécration de la notion d'usage de l'eau. Elle précipite la ressource entièrement dans la sphère marchande et ce, sur l'ensemble du territoire national. Ainsi l'Etat, propriétaire de l'usage et libre dépositaire de son utilisation, participe comme acteur à la vie économique. Face au débat public/privé, une solution mixte semble avoir été trouvée. L'Etat s'approprie l'usage mais en laisse la concession à des compagnies privées reconnaissant ainsi les intérêts locaux et des propriétaires riverains. Cette solution laisse malgré tout l'Etat en position de domination ;

- administrative et institutionnelle, dans la mesure où désormais à cette époque un seul corps d'ingénieurs, celui des Ponts et Chaussées, domine de façon hégémonique l'expertise et l'ensemble des processus décisionnels concernant les usages de l'eau, marginalisant les services techniques du Ministère de l'Agriculture. (Haghe, 1998, p. 531) ;

- territoriale, avec le renforcement de la départementalisation. L'Etat reconnaît l'existence d'un pouvoir local mais encadre ce mouvement dans des territoires où son autorité (administrative, juridique, technique) s'exerce fortement. La mise en place d'un découpage par bassin reste encadrée dans le sens où les régions économiques ou hydrographiques reposent fortement sur les découpages départementaux.

La gestion intégrée des usages de l'eau à l'échelle d'un bassin versant dont s'inspire notre organisation actuelle prend ses racines dans le contexte politico-économique marqué par la création de la Compagnie Nationale du Rhône (CNR) le 27 mai 1921․ Il faudra cependant attendre près de quarante ans pour qu'elle soit effective. En effet, les aspirations initiales qui motivèrent le retour $d u$ bassin versant ne seront pas suivies d'effets. Les aspirations régionalistes ne pourront contenir la pensée centraliste jacobine comme « en témoigne le faible impact de la CNR sur l'économie locale » (Béthemont, 1999, p. 230).

Les croisements entre organisation, administration $\mathrm{du}$ territoire et bassin hydrographique nous renseignent, par le contenu des débats et des orientations suivies, sur les enjeux relatifs à l'identification, au découpage et à l'articulation des territoires de l'eau ainsi que sur la recomposition des rapports entre "sphère publique ", "sphère privée » et "sphère communautaire ». Des stratégies s'affrontent pour le contrôle des eaux courantes et pour des raisons à la fois économiques, politiques, institutionnelles et scientifiques une territorialisation hydraulique à visée rationaliste s'impose progressivement d'abord à l'échelle locale puis à l'échelle régionale. La reconnaissance institutionnelle intervient dans les années 1960 en France où le bassin versant devient le cadre de la politique de l'eau. Depuis quelques années, cette forme de territorialisation à partir d'une approche fonctionnaliste et dans ce cas précis d'une définition naturelle de l'espace, n'est pas une exception même si elle s'est généralisée à toutes les échelles (du local à l'international). Désormais, la territorialisation touche en effet l'ensemble des politiques publiques, même les plus régaliennes (police, justice, éducation). 
31 Depuis une dizaine d'années, dans le cadre de leur l'analyse, la territorialisation est définie comme telle: elle consiste à afficher des priorités localisées (un principe de " proximité » et de "flexibilité ») tout en assurant une cohérence entre les actions des différents partenaires (principe d'unité) notamment à l'aide de contrat (Espain, 2004). Dans un contexte de décentralisation, engagé dans les années 1980 et fortement remis en débat depuis les années 1990 avec l'intervention politique plus prégnante de l'Union européenne, la question de l'articulation ou de la coordination de ces politiques devenues «territoriales » constitue donc un enjeu pour les pouvoirs publics. La dernière décennie tend à montrer que des blocages et des résistances fortes s'établissent entre ces politiques et les acteurs qui les portent. Le territoire se trouve instrumentalisé.

Le contexte et les objectifs ont donc sensiblement changé pour la territorialisation. Dans le domaine de l'eau, s'il s'agit toujours de participer à l'organisation d'un territoire, aujourd'hui dans le cadre d'un bassin versant, la territorialisation doit désormais (de manière paradoxale) composer et tenter de "réguler » des territorialisations multiples et non restreintes au domaine de l'eau. Ces dernières proviennent entre autres d'une concurrence accrue et de plus en plus conflictuelle entre les multiples fonctions du territoire (productives, touristiques, agricoles, urbanistiques, environnementales, etc.) pour l'utilisation et la gestion de ses ressources (fonciers, ressources en eau, biodiversité, paysages, etc.). Ces territorialisations multiples encouragent les processus d'appropriation des ressources d'autant plus aigus que pour certains territoires, dans un contexte d'affirmation et de reconfiguration des pouvoirs locaux, l'eau n'est plus uniquement un support mais un facteur de développement.

La gestion territoriale constitue une innovation dans sa déclinaison et son organisation institutionnelle et politique à l'échelle du bassin avec les célèbres Agences de bassin devenues Agence de l'eau. Cette étape marque la reconnaissance du bassin versant comme territoire opérationnel de gestion à l'échelle régionale. Deux grandes périodes marquent l'évolution et les missions des organismes de bassin. La régionalisation fut difficile à mettre en place à cause du discrédit hérité de Vichy. La planification avec l'émergence de la région comme " problème » joua un rôle décisif.

Les premières formes d'une organisation par bassin versant se trouvent placées dans une perspective de développement et d'aménagement du territoire national assis sur des bases régionales. Elles émergent dans la période de l'entre-deux-guerres dans un contexte de relance économique fortement impulsée par l'Etat, via une politique volontariste de grands travaux. Le mouvement s'amplifie dans la décennie 1950-1960. Les exemples les plus emblématiques, inspirés du modèle de la Tennessee Valley Authority (TVA) aux Etats Unis, sont la CNR ou les Sociétés d'Aménagement Régional (SAR) pour la France ${ }^{9}$. Le développement régional devient le fer de lance de la politique d'aménagement du territoire dont le décret du 30 juin 1955 consacre la régionalisation du Plan.

Dans le cadre d'une politique de l'offre, l'objectif est alors la maîtrise hydraulique des flux par l'application d'une logique équipementière et notamment la construction de barrages dont la finalité est d'assurer la production d'eau à des fins multiples (agricole, énergétique ou de navigation). Centrée sur un acteur dominant, l'Etat et cloisonnée par types usages, cette gestion par filière (Barouch, 1989) repose sur une organisation appelée "mono acteur / unifonction" (Puech, Boisson, 1995). Territorialisation de type "descendante ", elle demeure cloisonnée dans des logiques propres à chaque usage et génère des dynamiques pouvant être en contradiction avec les dynamiques globales assurant la reproductibilité qualitative et quantitative de la ressource. Elle se différencie 
ainsi d'une approche plus contemporaine de la gestion territoriale dans le sens où elle ne participe pas à une prise en considération transversale des problématiques de l'eau rendue nécessaire par la mutation des usages et par une ouverture limitée et sélective du processus de décision aux acteurs locaux. Les limites financières, économiques, sociales et environnementales du modèle et les besoins de changement sont très vite apparus inévitables. L'inertie du système portera ce type de développement basé sur l'exploitation des ressources en eau jusque dans les années 1980 mais la rupture intervient au début des années 1960.

La période de reconstruction est marquée par une intense activité économique, urbanistique et démographique qui accroît la demande et la pression sur la ressource en eau. Il devenait urgent de trouver le moyen de financer le développement et l'entretien des infrastructures nécessaires au maintien de l'activité économique mise en place dans le cadre de la région. A l'instar de l'espace économique, l'espace de la gestion de l'eau devient « une région » dans le sens que lui confère la science régionale, c'est-à-dire un espace à problèmes qu'il faut gérer (Benko, 1998). Ce vaste mouvement de réorganisation et de mise en cohérence des espaces de l'aménagement et du développement régional se concrétise par la création en 1963 de la Délégation à l'Aménagement du Territoire et à l'Action Régionale (DATAR). L'essentiel de son activité s'inscrit dans un objectif de cohésion qui en fait une instance de proposition, d'arbitrage et de synthèse des politiques ministérielles sectorielles. La DATAR donne naissance un an plus tard aux Agences de bassin (Barraqué, 1997). Si la logique hydrologique a largement prévalu dans le découpage des Agences, la réalité est beaucoup plus complexe (efficacité environnementale, efficience économique, dimension internationale et régionale, nécessité d'incorporer les grands corps de l'Etat) (Barraqué, 1999a).

Les Agences de l'eau telles que nous les connaissons aujourd'hui sont instituées par la loi sur l'eau de $1964^{10}$ et constituent la seconde évolution. Présentées comme la première étape de la décentralisation de la gestion de l'eau en France, elles naissent quand même en pleine période de planification et détonnent donc par rapport au paysage institutionnel français de l'époque. Elles ont pour objectif de lutter contre la pollution, les pénuries localisées, les conflits d'usage dus à une pression quantitativement et qualitativement accrue suite à la mutation de la société française après la Seconde Guerre mondiale. De fait, le véritable principe novateur est la reconnaissance d'un nouveau découpage qui identifie six grands bassins ${ }^{11}$ sur le territoire national, chacun d'entre eux abritant une Agence et un Comité de bassin (Art. 14 et 13) qui ouvre le dispositif en direction des collectivités territoriales et les usagers du bassin (Nicolazo, 1997). Le mode de financement de cette politique est également « original ». Chargées de la collecte et de la distribution des redevances et de l'application du principe pollueur/payeur, les Agences de bassin sont l'outil d'exécution de la politique définie par les Comités. Cette particularité de prélever un impôt, sans contrôle du Trésor Public et du Parlement a été inconstitutionnelle jusqu'en décembre 2001 date à laquelle le Parlement a recouvré le pouvoir de décider de la fixation des redevances perçues par les Agences. L'enjeu n'est pas mince puisque cette particularité assure l'autonomie financière des Agences vis-à-vis du budget de l'Etat. Bernard Barraqué (1997, p. 188) rappelle que si le système « continue de fonctionner, c'est qu'il remplit un rôle essentiel dans la modernisation des politiques publiques [...] avec le temps elles apparaissent de plus en plus comme des institutions décentralisées ». Bien qu'encore en cours de discussion au Parlement, les récents débats et les orientations 
financières et organisationnelles prises autour de la nouvelle loi sur l'eau laissent à penser à une reconsidération (temporaire ?) de cette position.

Schéma $n^{\circ} 3$ : Aménagement du territoire et gestion de l'eau. Une logique d'accompagnement.

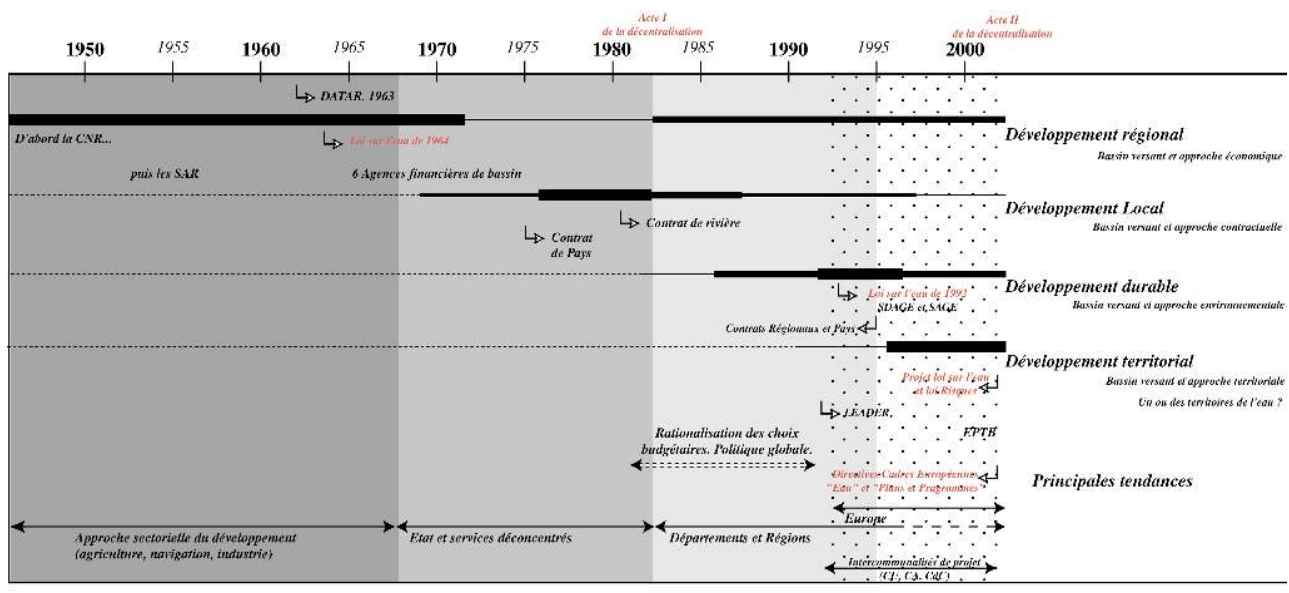

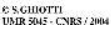

[Agrandir le graphique]

Les années 1990 constituent de ce point de vue un véritable tournant et ce aux différentes échelles. Les politiques de l'eau intègrent de plus en plus fortement « l'impératif milieu » ce qui bouleverse les approches en terme de gestion, désormais articulée autour des références systémiques et de bassin versant. De manière concomitante, on assiste à la recomposition des paradigmes du développement et à la prise en charge de ce dernier par les collectivités territoriales aux prérogatives accrues avec les lois de décentralisation (cf. schéma 3). A cela s'ajoute le rôle considérable joué par l'Union européenne (multiplication des politiques environnementales, impacts des Directives relatives à la gestion qualitative et quantitative de l'eau (plus de trente entre 1975 et 2000), instaurations de zonages...) et par les bailleurs de fonds internationaux (PNUE, FAO, B anque Mondiale, FMI, ETC.) qui associent le bassin versant aux principes de la «bonne gouvernance » (Ghiotti, 2004).

L'évolution des politiques publiques en matière d'aménagement, de développement territorial et de gestion de l'eau s'est effectuée de manière conjointe autour des principes de la trans-sectorialité et de la trans-territorialité. Ce changement de finalités s'accompagne d'une redéfinition des logiques et des territoires d'intervention. Les objectifs visent à une meilleure répartition des services, des infrastructures mais également des compétences et des rôles des différents acteurs de l'aménagement dans des cadres spatiaux modifiés, plus en phase avec les dynamiques économiques, sociales et environnementales qui les façonnent. Les Schémas Directeurs d'Aménagement et de Gestion des Eaux (SDAGE), les Schémas d'Aménagement et de Gestion des Eaux (SAGE), les Pays, les Parcs Naturels Régionaux, sont autant de nouveaux découpages territoriaux qui tentent de répondre à ces nouvelles problématiques. La loi sur l'eau de $1992^{12}$, la LOADDT de $1999^{13}$ dite loi "Voynet ", se construisent ainsi autour de référentiels communs, la durabilité, la globalité, la territorialisation de l'action et la contractualisation (entre collectivités, Etat et Union européenne). L'approche par bassin versant a donc accompagné le vaste mouvement de décentralisation de la politique de l'eau engagée au début des années 1980. Le SAGE, par sa dimension réglementaire (mais non obligatoire mise à part décision préfectorale) est alors venu compléter le dispositif en place relatif à 
la gestion et l'aménagement des rivières assuré jusqu'alors par la contractualisation avec les contrats de rivières (Brun, 2003).

Tableau $\mathrm{n}^{\circ} 1$ : Territorialisation des politiques de l'eau : des formes multiples et un bilan mitigé

\begin{tabular}{|l|c|}
\cline { 2 - 2 } \multicolumn{1}{c|}{} & \multicolumn{1}{c|}{$\begin{array}{c}\text { Les SAGE en France } \\
\text { (situation au 29/04/2005) }\end{array}$} \\
\hline $\begin{array}{l}\text { Mise en } \\
\text { œuvre }\end{array}$ & $\begin{array}{c}24 \text { (dont 9 pour le bassin Rhône- } \\
\text { Méditerranée-Corse, soit 37.5\%) }\end{array}$ \\
\hline Elaboration & 69 \\
\hline Instruction & 16 \\
\hline Emergence & 18 \\
\hline Total & 127 \\
\hline
\end{tabular}

\begin{tabular}{|c|c|}
\hline $\begin{array}{c}\text { Les contrats de rivière en France } \\
\text { (situation au 01/01/2005) }\end{array}$ & \\
\hline 61 & Contrats achevés \\
\hline 56 & $\begin{array}{c}\text { Contrats signés en } \\
\text { cours d'exécution }\end{array}$ \\
\hline 46 & $\begin{array}{c}\text { Contrat en cours } \\
\text { d'élaboration }\end{array}$ \\
\hline $\begin{array}{l}163(\text { dont } 81 \text { pour le bassin Rhône-Méditerranée-Corse, } \\
\text { soit 49.7\%) }\end{array}$ \\
\hline
\end{tabular}

Source : Ministère de l'Ecologie et du Développement Durable, 2005.

Les politiques de l'eau sont progressivement devenues de moins en moins sectorielles et de plus en plus globales. Leur territorialisation se caractérise de la manière suivante. D'abord, ce processus a très largement favorisé le fait que la définition de la problématique et du périmètre, le règlement et le financement de la gestion soient une construction sociale et politique au niveau local entre les différents usagers. Le contenu et les objectifs poursuivis tendent à évoluer depuis une dizaine d'années pour intégrer des problématiques de développement. Dans ce sens, les finalités territoriales des politiques de l'eau ne sont plus seulement l'application de politiques sectorielles. Elles visent à s'inscrire dans des projets de développement territorial, cherchant à devenir un élément fédérateur pour les acteurs locaux et à assurer une relative mise en cohérence des politiques liées à l'eau et à l'aménagement. En effet, les gestionnaires de l'eau essaient d'influer le plus en amont possible sur les modalités d'occupation de l'espace et donc sur les politiques touchant les domaines de l'agriculture, de l'urbanisme ou encore du tourisme (Narcy, 2004). Des territoires de gestion plus « englobant », type bassin versant, sont ainsi proposés dans une logique territoriale. Ces nouvelles modalités de gestion de l'eau et des territoires entrainent des confrontations entres acteurs que les démarches participatives et la concertation, certes nécessaires, sont loin de pouvoir épuiser.

Frédéric Giraut (1999, p. 41) définit la logique territoriale comme « supposant un maillage et visant à la mise en place ou au soutien de projets d'aménagement et de développement définis dans un périmètre circonscrit et approprié. Elle est une dimension de l'aménagement du territoire quasi originelle; son poids relatif n'a cessé de s'accroitre tandis que ses références et ses objectifs 
évoluaient considérablement ». Selon l'auteur, elle tend à succéder aux logiques sectorielles et spatiales qui l'accompagnent dans toute politique d'aménagement: "c'est le poids respectif de chacune d'elles qui permet d'évaluer ou d'apprécier l'économie générale de la politique proposée ou mise en ceuvre ». L'évolution de ces trois logiques dans le temps et dans l'espace se remarque particulièrement dans la modification des périmètres destinés à la gestion de l'eau (cf. schéma n4).

La loi sur l'eau de 1992 poursuit ainsi la territorialisation de la gestion de l'eau et l'inscrit dans une dimension locale avec les SDAGE et les SAGE. Souvent présentée comme une nouvelle phase de la décentralisation de la politique de l'eau, cette étape correspond également à un retour de l'Etat et de la planification dans ce domaine (Aspe, 1998). Face à l'augmentation des besoins, l'idée pour l'Etat était de gérer l'eau au plus près des consommateurs et des usagers, de jouer le jeu de la décentralisation tout en mobilisant les financements des collectivités locales sur des politiques nationales. Ainsi, à l'origine du projet du Ministère de l'Environnement n'était prévue que la création de structures locales, les SAGE. Ces derniers et donc les acteurs locaux chargés d'animer ce nouveau territoire dans le cadre d'une Commission Locale de l'Eau, CLE ${ }^{14}$ étaient alors en liens directs avec l'administration centrale aux prérogatives renforcées. Ce projet a rencontré l'opposition des Agences pour qui il était inconcevable que se crée un territoire de l'eau en dehors de leur contrôle (Vieillard-Coffre, 2001). Une solution de compromis fut trouvée avec la création du SDAGE. Le SDAGE vise à l'échelle du territoire des Agences (il $\mathrm{y}$ a donc un seul SDAGE par Agence), à planifier et à rendre cohérentes les politiques qu'elles définissent. Avec cette disposition, les SAGE, élaborés par les acteurs locaux, doivent ainsi être compatibles avec les orientations définies par le SDAGE et donc être en conformité avec la politique des Agences. Ces dernières ressortent donc légitimées et renforcées puisque le SDAGE s'impose au SAGE.

La mise en œuvre des SAGE et des CLE à l'échelle locale intègre et interfère avec les logiques politiques et territoriales déjà existantes dans la mesure où, décentralisation oblige, les élus locaux sont amenés à s'investir de plus en plus dans les politiques de l'eau. Elle participe ainsi à la création d'une gouvernance de bassin nécessitant la recomposition du pouvoir local et ce pour trois raisons :

- la CLE constitue un nouveau lieu de concertation, différent dans sa composition et son fonctionnement des « scènes " politiques traditionnelles. L'intégration de nouveaux acteurs modifie les rapports de force politiques et participe à une autre lecture des problématiques d'aménagement et de développement liées à un territoire donné.

- faute de posséder la maîtrise d'ouvrage et de budget propre, la CLE doit s'appuyer sur une structure intercommunale porteuse pour fonctionner, ce qui modifie les rapports entre ces structures et donc le jeu politique entre « leaders ».

- enfin, la CLE, dans le cadre de la gestion globale et territoriale de l'eau est très souvent amenée à intervenir dans les domaines de la lutte contre les inondations et les pollutions, les débits d'étiage, le maintien des paysages, etc. En intervenant sur des espaces et des thématiques en lien avec d'autres dynamiques en termes de développement et d'aménagement (agricole, urbain, industriel, touristique), la CLE se trouve confrontée avec d'autres acteurs et d'autres logiques. Les gestionnaires de l'eau et ceux du développement se retrouvent donc dans une relative concurrence (thématique et spatiale) poursuivant les mêmes finalités de développement territorial et travaillant sur des échelles territoriales d'intervention relativement comparables mais sur des périmètres souvent peu articulés. 


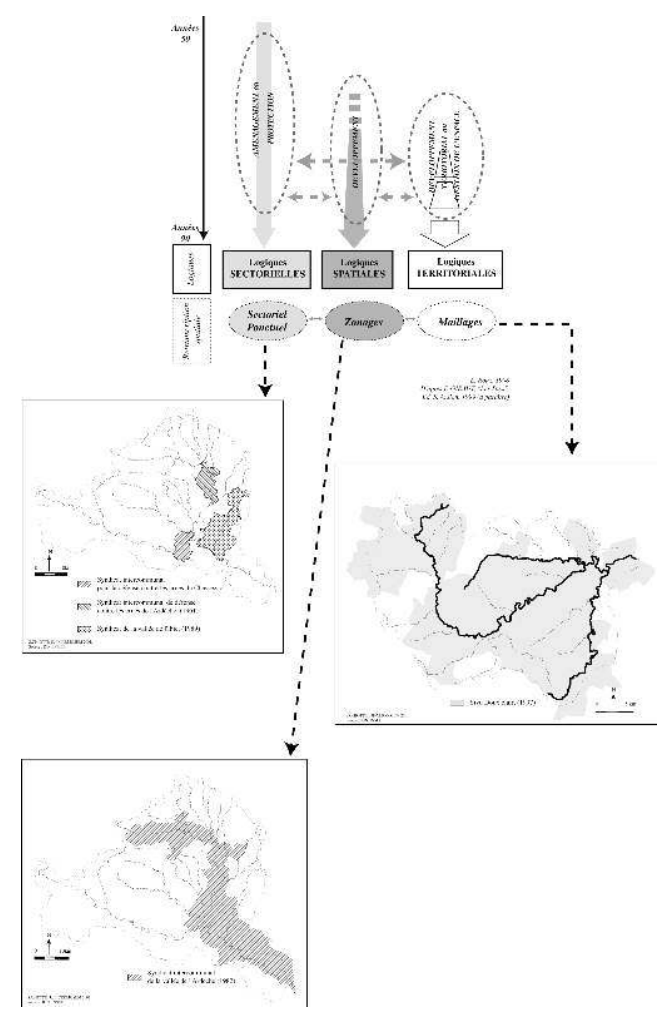

La multiplicité des centres de pouvoir et la diversité des échelles d'intervention participent à la généralisation de la contractualisation. La décentralisation avec la reconnaissance du Département, de la Commune et de la Région comme collectivités territoriales, ouvre le jeu d'acteurs à d'autres partenaires qui deviennent ainsi numériquement plus importants. Certaines grandes villes et Départements ont pu mettre en place grâce à des moyens financiers et humains conséquents des services administratifs et techniques dotés d'une capacité de réflexion et d'action, les rendant progressivement autonomes voire concurrents des services de l'Etat. Cette concurrence n'a cessé de s'accentuer avec la mise en place des Régions. L'Etat ne bénéficie plus du monopole de la norme, de la capacité d'expertise et de l'intérêt général (Duran, Thoenig, 1996). L'organisation verticale par filière se voit complétée d'une dimension horizontale tant la gestion des problèmes et des situations devient complexe et ne se contente plus d'une prise en charge et d'une résolution centralisée; comme le rappelle Emmanuel Négrier (2001, p. 30), « la définition du problème fait problème » dans tous les domaines «et l'identification des solutions repose sur une négociation de plus en plus complexe où plusieurs catégories d'intervenants (experts d'Etat, élus locaux, administrateurs territoriaux, intérêts privés, etc.) disposent d'une part de légitimité ».

Cette nouvelle forme de gouvernement local ne constitue d'ailleurs pas une exception française, puisque Bernard Barraqué (1999b) précise que, dans le domaine de la gestion de l'eau notamment, cette pratique s'est étendue à l'ensemble des pays européens tant dans le domaine des contrats de rivière que dans celui de l'eau potable et de l'assainissement. Concernant ce dernier exemple, l'intégration de nouveaux acteurs peut avoir des conséquences significatives surtout quand les rapports de force entre acteurs sont 
inégaux. C'est ainsi que dans les années 1980, gestion de l'eau et décentralisation ont conduit nombre de communes à déléguer au secteur privé, la gestion du secteur de l'eau potable et de l'assainissement.

Tableau $\mathrm{n}^{\circ} 2$ : Evolution de la participation financière des différents acteurs au contrat de rivière du Doux (France, Région Rhône-Alpes, Ardèche).

\begin{tabular}{|c|c|c|c|c|c|c|c|c|c|c|}
\hline & \multirow{2}{*}{$\begin{array}{c}\begin{array}{c}\text { Contrat de } \\
\text { rivière (1) }\end{array} \\
1993-1995\end{array}$} & \multirow{2}{*}{$\begin{array}{c}\text { Contrat de } \\
\text { milieu (2) } \\
1997-2001\end{array}$} & \multicolumn{2}{|c|}{ Volet A } & \multicolumn{2}{|c|}{ Volet B } & \multicolumn{2}{|c|}{ Volet C } & \multicolumn{2}{|c|}{ Volet D } \\
\hline & & & 1 & 2 & 1 & 2 & 1 & 2 & 1 & 2 \\
\hline UE & $31 \%$ & $10 \%$ & $28,5 \%$ & $8 \%$ & $28,7 \%$ & $11 \%$ & $28 \%$ & $9 \%$ & $50 \%$ & $25 \%$ \\
\hline Etat & $1,5 \%$ & - & - & - & $1,3 \%$ & - & $10 \%$ & - & - & - \\
\hline Région & $10 \%$ & $15 \%$ & $8,5 \%$ & $13 \%$ & $-\%$ & $7 \%$ & $7 \%$ & $21 \%$ & $30 \%$ & $16 \%$ \\
\hline Agence de l'eau & $25 \%$ & $22 \%$ & $27,7 \%$ & $19 \%$ & $50 \%$ & $33 \%$ & $25 \%$ & $37 \%$ & - & $4 \%$ \\
\hline C.Général 07 & $11 \%$ & $20 \%$ & $13 \%$ & $25 \%$ & $13,4 \%$ & $6 \%$ & $8 \%$ & $12 \%$ & - & $18 \%$ \\
\hline Syndicat & $21,5 \%$ & $33 \%$ & $22,3 \%$ & $35 \%$ & $6,6 \%$ & $43 \%$ & $22 \%$ & $21 \%$ & $20 \%$ & $37 \%$ \\
\hline
\end{tabular}

Source: S. Ghiotti., 2001, p. 333.

littéralement fait voler en éclats la répartition des compétences par blocs. Les nouvelles collectivités territoriales, si tant est qu'elles disposent des moyens nécessaires, sont susceptibles d'intervenir dans n'importe quel domaine en définissant des politiques et en multipliant les procédures. L'exemple du financement des contrats de rivière l'atteste comme il témoigne du désengagement financier de l'Etat en direction des collectivités (cf. tableau $n^{\circ} 2$ ). Ainsi, la dilution de l'action publique territoriale se double d'une sévère compétition entre institutions pour l'appropriation et le contrôle du ou d'un territoire, vu comme un moyen d'asseoir leurs politiques, d'affirmer leur légitimité et leur existence (au besoin d'affaiblir la collectivité voisine). Ces luttes sont d'autant plus intenses que les territoires en question sont « stratégiques » et/ou possèdent une forte charge symbolique et/ou naturelle et que les institutions sont en mal de reconnaissance ou remises en cause. En outre, la recherche de la position dominante se trouve largement encouragée par les nombreuses ressources et subsides auxquels elle donne droit mais également par le statut qu'elle confère au sein du réseau d'acteurs. Derrière cette précision, il est possible de regrouper bon nombre de configurations. Cependant, les rivalités entre Département, Région et Union européenne semblent particulièrement représentatives. Ces différents acteurs se livrent une véritable concurrence par politiques territoriales interposées pour le contrôle des territoires ruraux à forte dominante environnementale. Le territoire de gestion de l'eau devient un enjeu accentuant les interférences et les conflits entre les projets et les collectivités qui les portent. 
Carte $n^{\circ} 1$ : La gestion de l'eau par bassin : territorialisation ou déterritorialisation ?

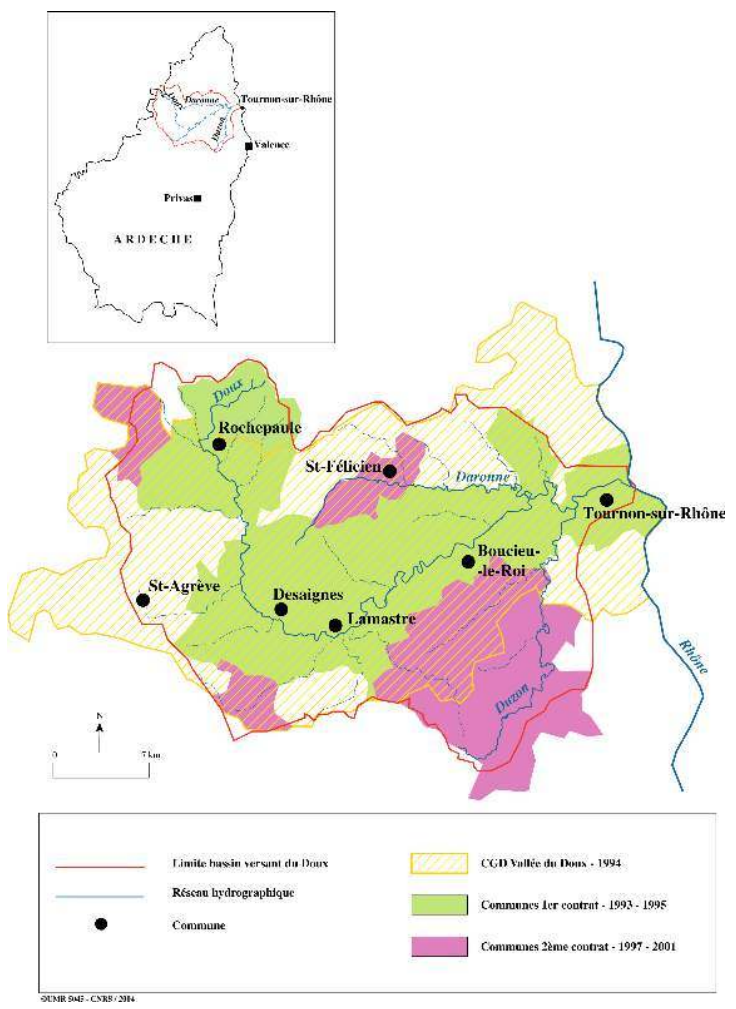

50 [Agrandir le graphique : Ghiotti_annexe 3.]

51 Au sein de cette complexité territoriale, le découpage par bassin versant, légitimé à toutes les échelles comme le territoire de la gestion de l'eau, se trouve mobilisé par différents acteurs. A l'instar d'autres découpages, le bassin versant se trouve complètement inséré dans des dynamiques de recomposition territoriale mais l'observation fait apparaître trois contextes particuliers dans lesquels le bassin versant est mobilisé. Les trois exemples rencontrés ne sont pas exclusifs les uns des autres et peuvent se retrouver à différents stades dans les dynamiques de développement territorial.

52 - La première situation rencontrée s'apparente à ce que l'on peut appeler «l'optimum territorial ». Volontairement provocatrice par son titre, cette configuration s'inscrit dans une " prospective ", voire une solution envisageable pour une territorialisation forte de la politique de l'eau où l'eau facteur de développement s'intègre très en amont des projets de développement et d'aménagement du territoire. Le choix et la construction du territoire s'effectuent à l'échelle locale (adaptabilité et proximité) tout en essayant de se donner les moyens d'assurer une certaine cohérence entre les différentes politiques (entre « local » et « global », dimension « verticale » et « horizontale » entre les différents acteurs). L'Ardèche Méridionale par exemple tend, en effet, à montrer que, même à l'échelle d'un grand bassin, un découpage s'inspirant des limites du bassin hydrographique est " pertinent ». Nous irions même jusqu'à dire, compte tenu des enjeux forts sur le sud Ardèche (tourisme, inondation, agriculture urbanisation, pollution), que le bassin versant dans le cadre d'un Schéma d'Aménagement et de Gestion des Eaux (SAGE ) serait un territoire capable de dépasser les antagonismes et devenir une maille opérationnelle. Gérer un territoire structuré économiquement et socialement autour 
d'une rivière permet d'élargir la problématique et d'intégrer d'autres logiques susceptibles de mobiliser un plus grand nombre d'acteurs.

- Deuxième configuration, l'eau est mobilisée comme facteur de solidarité territoriale et/ ou vecteur d'intégration, dans des procédures qui valident des espaces de mobilisation plus vastes et structurés à une autre échelle. Le bassin versant est considéré ici comme une plate-forme d'articulation, où les politiques contractuelles liées à l'eau assurent un rôle de complémentarité et de cohérence territoriales,

54 - La troisième situation témoigne d'une lutte institutionnelle relative au partage et au contrôle des compétences pour le développement et l'aménagement du territoire. Elle s'effectue entre une maille départementale remise en cause, une Région en quête de légitimité par un ancrage territorial, l'Union européenne dont l'appui au développement local se fait de plus en plus fort, via les Régions, mais aussi de manière autonome, et l'Etat en marge, cantonné dans un rôle de proposition avec la territorialisation de certaines politiques sectorielles (Giraut, Vanier, 1999).

55 La territorialisation de la politique de l'eau se décline en plusieurs types de territorialisation et participe clairement à la complexité territoriale en augmentant les possibilités de choix pour réaliser les stratégies spatiales des différents acteurs. A l'origine censé favoriser la cohérence territoriale, le processus ne conduit-il pas au mieux à différencier les territoires, au pire à les fragmenter?

Par rapport aux multiples configurations rencontrées, il est intéressant de noter que la " vieille » échelle départementale avec le Conseil Général tire son épingle du jeu et trouve dans le cadre de la décentralisation les moyens de moderniser son action. La stratégie territoriale de développement de certains Conseils Généraux se base essentiellement sur leur aptitude à capter ces territoires "d'intercession $»^{15}$ dont relève le bassin versant. Comme le rappellent Daniel Béhar et Philippe Estebe (2000, p. 5), "cette fonction d'intercession devient aussi essentielle que celle d'accès aux ressources financières. Bien souvent d'ailleurs, c'est la position d'intercesseur qui conditionne l'accès aux ressources, bien plus que la position institutionnelle ». Dans ce contexte, on comprend mieux pourquoi certains Conseils Généraux (exemple de l'Ardèche, de l'Hérault, du Gard notamment) en jouant la carte de la proximité et de la solidarité développent l'approche par bassin versant par un relatif contrôle et pilotage des contrats de rivière et des SAGE (au moins dans la période initiale). Ce territoire leur permet d'accéder à cette fonction et ainsi de rivaliser sur le champ du développement avec l'Europe (projets LEADER I, II, +), mais surtout avec la Région qui, dans le cas de Rhône-Alpes par exemple, favorise les CODRA (Contrat de Développement de la région Rhône-Alpes). Si cette volonté politique est visible dans le domaine de l'eau, elle se retrouve également dans celui de l'électricité (Poupeau, 2004) voire dans le domaine urbain (Ile-de-France). Bon nombre d'exécutifs départementaux prennent des postures volontaristes pour les deux premiers domaines dans la mesure où ils consolident des positions anciennes. En effet, l'eau et l'électricité ont constitué deux domaines de base de l'intervention départementale, pris en charge d'abord par l'intercommunalité de « service » puis par l'intercommunalité de " projet » dans le cadre du développement et de l'aménagement rural. De plus, au niveau institutionnel, l'échelle départementale possède des capacités techniques, financières et administratives qui en font un acteur souvent incontournable (DDAF, DDE, DDASS, services du Conseil Général, etc.). L'IFEN (2002) rappelait d'ailleurs que sur la période 1996-1999, les Conseils Généraux ont consacré 4,72 milliards d'euros à l'environnement contre 948 millions pour les Conseils Régionaux. En outre, si ces derniers consacrent $33,4 \%$ de leurs dépenses 
environnementales à l'eau, les premiers y participent à hauteur de 65,3\% (IFEN, 2003). Les nouveaux transferts de compétence et la suppression du FNADE pour les départements métropolitains (mais son remplacement par un fonds départemental pour l'alimentation en eau et l'assainissement) réduiront-ils ces écarts?

Même s'il est encore trop tôt pour tirer des conclusions face à des processus en cours, des pistes peuvent être dégagées et des éléments d'interprétation avancés. Une fois n'est pas coutume, les récents débats sur la poursuite de la décentralisation conjugués à la réforme de la politique de l'eau ont donné un nouvel élan aux relations entre les collectivités. Ces dernières ont alors élaboré un certain nombre de projets ${ }^{16}$ et émis des demandes de transfert dans le cadre d'une nouvelle répartition des compétences entre les différents niveaux et de l'expérimentation. Deux rapports officiels serviront de support à la compréhension des positionnements des différents acteurs par rapport à la politique de l'eau (Launay, 2003 ; Flory, 2003).

Deux tendances se dessinent clairement. Le rapport Flory propose de maintenir le système actuel des Agences avec son mode de financement actuel, en intégrant toutefois des évolutions sensibles en réponse aux critiques émises par de nombreux rapports (Commissariat général du plan, 1997 ; Cours des comptes, 2003). Le rapport propose le passage des redevances devant le Parlement, la modification de leur calcul, le développement de la transparence et de la démocratie et enfin « la reconnaissance » de la légitimité des départements dans le domaine de l'alimentation en eau potable et de l'assainissement. En cela, il rejoint d'autres analyses faites sur le sujet, notamment celles effectuées par le Commissariat général du Plan à propos de la préservation de la ressource en eau destinée à l'alimentation humaine (Commissariat général du Plan, 2001, p. 32). La remise en cause du système est beaucoup plus développée dans le rapport Launay. Il reconnaît et valide aussi la pertinence du transfert (compétence et financement) de l'alimentation en eau potable et de l'assainissement au titre de la solidarité (financière et territoriale) vers le département (Conseil Général). Mais ses propositions vont bien au-delà pour s'insérer plus fortement dans la lutte contre les inondations mais surtout dans la gestion de la ressource, véritable chasse gardée des Agences de Bassin. Il réclame ainsi, pour ce faire, que soit reconnues pour les Etablissements Publics Territoriaux de Bassin (ЕРTB) des compétences en matière de mise en œuvre des actions à l'échelle des bassins versants ${ }^{17}$. En demandant la création d'un EPTB par commission géographique de bassin, les EPTB et les collectivités territoriales qui les portent se veulent en concurrents directs des Agences.

Pour la Région Bretagne mais aussi l'Alsace, les propositions visent à créer un Schéma Régional d'Aménagement et de Gestion des Eaux (SRAGE) qui se placerait entre le SDAGE et le SAGE. La question de la valeur juridique du SRAGE reste à établir de même que les relations entre ce dernier et les SAGE. Le Conseil Régional exercerait ainsi les fonctions de guichet unique, de planificateur (SRAGE) et de centralisateur des données. Bien sûr, il est réaffirmé « la prééminence du bipôle région-agence de l'eau » afin notamment de pouvoir bénéficier des compétences et des moyens humains de l'Agence. Cette volonté de regrouper à l'échelle régionale, compétence et informations, au nom de l'efficacité, conduit aussi les promoteurs du projet à envisager le rattachement de la délégation régionale de l'ADEME à la Région. Voulant par ces propositions lutter contre une « recentralisation rampante », les auteurs du manifeste proposent une recentralisation... mais à l'échelle régionale dont la mise en œuvre poserait de gros problèmes au vu des objectifs fixés par la Directive Cadre Européenne. Cette dernière remarque pose une autre 
question essentielle, celle de la maîtrise de l'expertise et montre que les débats ne sont pas tranchés en la matière. L'approche territoriale appliquée au domaine des politiques de gestion de l'eau complexifie énormément leur mise en œuvre et exige pour les collectivités responsables des moyens, des compétences et des savoirs-faire d'un haut niveau (juridique, technique, administratif, financier, management, "monitoring", " engineering ", etc.). Qui est à même d'exercer ces compétences? Un nouveau Corps d'ingénieur de l'environnement? Les orientations prises dans le cadre de la décentralisation semblent ne pas aller dans cette direction que la réorganisation des services déconcentrés de l'Etat à l'échelle régionale ne semble pas non plus en mesure d'infléchir. Pour le contrôle et l'exercice de l'expertise, une autre architecture semble se mettre en place. A l'opposition public/public («agronomes » / « Pont et Chaussées») de la période précédente se substitue un "partenariat " associant public / privé / réseaux d'associations et $\mathrm{ONG}$ spécialisées ${ }^{18}$, comme le laisse à penser l'exemple de la gestion des réserves naturelles en France (Petit, 2002) ou le cas de la réforme de la politique de l'eau au Liban (Ghiotti, 2003). Malgré tout, les questions de l'expertise et de la coordination territoriale restent ouvertes, dans le sens où demeurent très clairement posés deux problèmes : celui des rapports de forces entre ces catégories, d'évidence inégaux, et celui du contrôle démocratique de ces politiques territoriales, avec une expertise qui peut être complètement a-territoriale, et basée sur des intérêts économiques, financiers ou catégoriels.

La territorialisation de la gestion l'eau apparaît comme une forme d'organisation sociale ancienne et complexe, en lien direct avec les luttes politiques, institutionnelles, et scientifiques pour l'appropriation de la ressource et sa gestion. Les territoires de l'eau évoluent fortement dans ces échelles, ces objectifs et ces finalités entre le XIX ${ }^{\text {eme }}$ et le XX eme siècle, en lien étroit avec les différentes étapes de décentralisation. D'une organisation locale du partage de l'eau entre usagers sur des bases communautaires, la gestion territoriale de l'eau évolue vers un partage de la ressource entre usages au sein des problématiques touchant d'abord l'aménagement régional puis le développement territorial. En parallèle à cette évolution, les territoires de l'eau s'homogénéisent progressivement dans le cadre du bassin hydrographique puis du bassin versant. L'approche par bassin devient le cadre de la gestion territoriale de l'eau et le fondement du modèle français, dont l'expérience s'exporte et se généralise à l'échelle européenne et internationale.

61 Avec les années 1990 et la montée des ressources locales notamment environnementales dans le développement, l'approche par bassin apparaît comme une réponse à la complexité territoriale à l'œuvre, provoquée par la relance de la décentralisation, le dynamisme de la construction de l'Union européenne et la mondialisation. La territorialisation des politiques sectorielles de l'Etat et leur transfert vers les collectivités locales, en situation de concurrence, serait la dernière étape de la décentralisation. Au moins dans les discours, elle est voulue comme la condition de la modernisation, de la performance et de la légitimation des différentes institutions et des politiques qu'elle porte. Cependant les liens tissés entre eau et développement montrent que, en devenant un territoire politique, le bassin versant se trouve être rattrapé par une logique qu'il devait dépasser pour constituer une forme hybride au carrefour des influences politiques, administratives, socio-économiques et environnementales. Le territoire "problème " devient le territoire porteur de stratégies et de " potentialités ». Mais « les potentialités » et les ressources ne sont pas les mêmes selon les collectivités et les transferts de 
compétences prévus pourraient aggraver les disparités et les inégalités territoriales et ce malgré les systèmes de péréquation envisagés. Dans le même temps, d'autres collectivités demandent au titre de l'expérimentation, le transfert de la politique de l'eau ce qui suppose qu'elles possèdent les moyens suffisant et adéquats pour sa mise en œuvre. La territorialisation, en voulant s'adapter aux spécificités locales, ne constitue-t-elle pas une dilution voire une négation de l'action publique et de l'intérêt général ? L'Etat garant de ce même intérêt veut dans le contexte actuel se concentrer sur ses métiers et ses missions dites « de base ». L'intégration et la solidarité territoriale ne sont-elles pas justement au centre de ses missions?

\section{BIBLIOGRAPHIE}

Affeltranger B., Lasserre F., 2003, « La gestion par bassin versant : du principe écologique à la contrainte politique - le cas du Mékong ", La revue en sciences de l'environnement - VertigO, $\mathrm{n}^{\circ} 3$, Vol.4, 15 p. (décembre) (en ligne, avec le reste des articles de la revue, sur http:// www.vertigo.uqam.ca).

Aspe Ch., 1998, « Le retour de l'Etat et l'argumentaire scientifique dans les conflits pour l'eau », in Régulation de l'eau en milieu méditerranéen, risques et tensions, Territoires en Mutation, $\mathrm{n}^{\circ} 3$, mars, $\mathrm{pp}$. 83-94.

Aubriot O., Jolly G. (coord.), 2002, Histoires d'une eau partagée. Provence, Alpes, Pyrénées, Aix-en Provence, Université de Provence, 248 p.

Barouch G., 1989, La décision en miettes : systèmes de pensée et d'action à l'œuvre dans la gestion des milieux naturels, Paris, L'Harmattan, Coll. Logiques sociales, $237 \mathrm{p}$.

Barel Y., 1981, « Modernité, code, territoire », in Les Annales de la recherche urbaine, $\mathrm{n}^{\circ} 10 / 11, \mathrm{pp}$. 3-21.

Barraqué B., 1999a, «Forum IV », in : Cultures, Usages et Stratégies de l'eau en Méditerranée Occidentale, tensions, conflits et régulations, Marié M., Larcena D., Derioz P. (Dir.), Paris, L'Harmattan, Coll. villes et entreprises, pp. 535-543.

Barraqué B., 1999b, « Aperçu sur les politiques contractuelles de l'eau dans les pays de l'Union européenne ", in : La contractualisation, moyen d'approfondissement et de mise en cuvre de la politique de l'eau, Paris, Cercle Français de l'eau, Sénat, pp. 27-31.

Barraqué B., 1997, « Subsidiarité et politique de l'eau », in : Territoires et subsidiarité. L'action publique locale à la lumière d'un principe controversé, Faure A. (Dir.), Paris, L'Harmattan, coll. Logiques politiques, pp.165-201.

Béhar D., Estebe P., 2000, « Décentralisation ; l'acte II est déjà joué », in : L'Etat de la France. Un panorama unique et complet de la France 2000-2001, Politiques publiques et territoires, mai , 5 p.

Benko G., 1998, La science régionale, Paris, PUF, Coll. Que sais-je ?, n 3355, 126 p.

Berger L., 1998, Développement et ressources en eau dans trois vallées de la bordure orientale du Massif central (XIXème et XXème siècle) : la Turdine, le Gier et la Cance, Presses universitaires du Septentrion, Coll. Thèse à la carte, $583 \mathrm{p}$. 
Béthemont J., Faggi P., Zoungrana Tanga P., 2003, La vallée du Sourou (Burkina Faso). Genèse d'un territoire hydraulique dans l'Afrique soudano-sahélienne. Paris, L'Harmattan, $230 \mathrm{p}$.

Béthemont J., 2000, Géographie de la méditerranée, Paris, A. Colin, Coll. U Géographie, 313 p. Béthemont J., 1999, Les grands fleuves, entre nature et société, Paris, A. Colin, Coll. U Géographie, 255 p.

Billaud J-P., 1984, Marais Poitevin, Rencontres de la terre et de l'eau, Paris, L'Harmattan, 265 p.

Bonnaud L., 2000, « Aimé Thomé de Gamond (1807-1876) : a pioneer of the unachieved », in: Telling Lives, horne A. (edit.), Macmillan, pp. 335-351.

Broc N., 1991, Les montagnes au siècle des lumières, Paris, Editions du Comité des Travaux historiques et scientifiques, $299 \mathrm{p}$.

Commissariat général du Plan, 2001, La politique de préservation de la ressource en eau destinée à la consommation humaine, Rapport d'évaluation, Villet-Desmeserets F., (rapporteur général), Paris, La Documentation Française, 402 p.

Commissariat général du Plan, 1997, Evaluation du dispositif des Agences de l'eau, Rapport au Gouvernement, Paris, La Documentation Française, 215 p.

Cour des Comptes, 2003, « Chapitre VIII, Ecologie et développement durable », in : Rapport au Président de la République, suivi des réponses des administrations, collectivités, organismes et entreprises, pp. 315-387.

Debarbieux B., 1988, Territoires de haute montagne: recherches sur les processus de territorialisation et d'appropriation de l'espace en haute montagne dans les Alpes du Nord, thèse de géographie, Université Joseph Fourier - Grenoble I, 495 p.

Di Méo G., 1998, Géographie sociale et territoire, Paris, Nathan Université, Fac Géographie 320 p.

Di Méo G., 1994, "Patrimoine et territoire, une parenté conceptuelle", in : Méthodes et enjeux spatiaux, Espaces et Sociétés, n78, pp. 15-34.

Epstein R., 2004, « Après la territorialisation, la différenciation territoriale ? ", Pouvoirs locaux, $\mathrm{n}^{\circ}$ 63, pp. 35-42.

Drain M., 1998, Régulation de l'eau en milieu méditerranéen risques et tensions, Territoires en mutation, $\mathrm{n}^{\circ} 3$, mars, $263 \mathrm{p}$.

Duran P., Thoenig J-C., 1996, « L'Etat et la gestion publique territoriale », Revue Française de Science Politique, vol. $46, \mathrm{n}^{\circ} 4$, pp. 580-623.

Flory J-Cl., 2003, Les redevances des agences de l'eau. Enjeux, objectifs et propositions d'évolution dans la perspective de la réforme de la politique de l'eau, rapport au Premier Ministre et à la Ministre de l'Ecologie et du Développement Durable, octobre, 180 p.

Frapa P., 1997, « Restanques, faïsses ou banquettes : l'homme, l'eau et la pente », cité par Larcena D., 1999, «L'eau de collines », in : Cultures, Usages et Stratégies de l'eau en Méditerranée Occidentale, tensions, conflits et régulations, Marié M., Larcena D., Derioz P. (Dir.), Paris, L'Harmattan, coll. villes et entreprises, pp. 116-126.

Frémont A., 1976, La Région, espace vécu, Paris, PUF, 233 p.

Gagnon Ch., 1994, « La recomposition des territoires, développement local viable », Paris, L'Harmattan, Coll. Logiques sociales, $271 \mathrm{p}$.

Gautier E., 1996, « Trois cents ans d'aménagements fluviaux dans la vallée du Buëch : de la rivière redoutée à la rivière sacrifiée ", in : Buëch et patrimoine : patrimoine, facteur de développement? 
Association départementale de sauvegarde du patrimoine du Pays du Buëch et des Baronnies, actes du colloque de Serre 19-20 avril, pp. 41-55.

Ghiotti S., 2001, La place du bassin versant dans les dynamiques contemporaines du développement territorial. Les limites d'une évidence. Approche comparée en Ardèche et dans les Hautes-Alpes, thèse de géographie, Université Joseph Fourier, Grenoble I, Institut de Géographie Alpine, pp. 68-105.

Ghiotti S., 2004, «Le bassin versant en question. Le modèle français de gestion de l'eau et les limites de son application au Liban ", in Du Sud au Nord, les territoires au nom du développement, A ntheaume B., Giraut F. (Dirs.), Paris : IRD, (à paraître).

Ghiotti S., 2003, « Participation, développement durable et politique de l'eau au Liban. Quel nouvel ordre négocié localement pour quel développement? ", in : La gestion concertée dans les espaces naturels protégés de montagne, Montagnes Méditerranéennes, $\mathrm{n}^{\circ} 18, \mathrm{pp} .89-96$.

Ghiotti S., 2000, « Irrigation traditionnelle et gestion collective de la ressource en eau en Ardèche : les béalières ", in : Approches sociales de l'irrigation et de la gestion collective de l'eau. Démarches et expériences en France et dans le monde, Territoires en mutation, Rivière-Honegger A., Ruf T. (Dir.), n7-mai, pp. 91-99.

Giraut F., 1999, « LOADDT : le sacre de l'approche territoriale et la relégation de l'approche spatiale », in : Territoires et développement : le nouveau dispositif législatif au crible, Montagnes Méditerranéennes, $\mathrm{n}^{\circ}$ 9, pp. 41-43.

Giraut F., Vanier M., 1999, « Plaidoyer pour la complexité territoriale », in : Utopie pour le territoire : cohérence ou complexité ? Gerbaux F. (Dir.), Editions de l'Aube, Coll. Société et Territoire, pp. 143-172.

Goussot A., 2003, «Place de l'eau et de sa gestion dans la construction et le développement d'un territoire : le cas de la Bièvre-Valloire (Isère et Drôme, France) ", Revue de Géographie Alpine, $\mathrm{n}^{\circ} 2$, tome 91, pp. 41-55.

Gumuchian H., 1991, Représentations et aménagement du territoire, Paris, Economica, Coll. Anthropos, 135p.

Haghe J-P., 1998, Les eaux courantes et l'Etat en France (1789-1919), du contrôle institutionnel à la fétichisation marchande, Paris, Thèse de doctorat de l'Ecole des Hautes Etudes en Sciences Sociales, $649 \mathrm{p}$.

Honegger A., 1990, L'eau en Camargue. Contribution de la géographie culturelle à la définition de paramètres pour une gestion optimale de l'espace de l'eau, Espace rural, $\mathrm{n}^{\circ} 23,272 \mathrm{p}$.

Hérodote., 2003, Les pouvoirs locaux, l'eau, les territoires, Paris, La Découverte, n¹10, 188 p.

Jaglin S., 2003, « La participation au service du néolibéralisme ? Les usagers dans les services d'eau en Afrique subsaharienne ", in : Gestion de proximité et démocratie participative : les «nouveaux » paradigmes de l'action publique, Bacqué M-H., Syntomer Y. (Eds.), Paris, La Découverte. Lagarde L., 1998, « Philippe Buache (1700-1773), Cartographe ou géographe ? », in : Terre à découvrir, terres à parcourir. Exploration et connaissance du monde XII - XIX siècle », Lecoq D., C hambard A. (Dir.), Paris, L'Harmattan, pp. 147-166.

Lagarde L., 1987, « Philippe Buache, ou le premier géographe français (1700-1773) », Mappemonde, Montpellier, Reclus, $n^{\circ} 2$, pp. 26-30.

Launay J., 2003, La gestion de l'eau sur le territoire, Assemblée Nationale, Délégation à l'aménagement et au développement durable du territoire, rapport d'information $\mathrm{n}^{\circ} 1170$, novembre, $93 \mathrm{p}$. 
Lewis N., 2001, La gestion intégrée de l'eau en France : critique sociologique à partir d'une étude de terrain (Bassin Loire-Bretagne), Université d'Orléans, Thèse de Sociologie, $498 \mathrm{p}$.

Loinger G., Nemery J-C. (Dir.), 1998, Recomposition et développement des territoires...Enjeux économiques, processus, acteurs, Paris, L'Harmattan, Coll. Administration, Aménagement du territoire, $381 \mathrm{p}$.

Manceron V., 2003, La carpe, l'épis et le canard. Une société sous tensions entre terres et eaux (La Dombes), Thèse de doctorat en Ethnologie, laboratoire d'ethnologie et de sociologie comparative, Nanterre, 496 p.

Marié M., Larcena D., Derioz P. (Dir.), 1999, Cultures, Usages et Stratégies de l'eau en Méditerranée Occidentale, tensions, conflits et régulations, Paris, L'Harmattan, Coll. villes et entreprises, 543 p.

Marié M., 1983, « Pour une anthropologie des grands ouvrages. Le canal de Provence », Les Annales de la recherche urbaine, $\mathrm{n}^{\circ} 21$, pp. 5-35.

Marié M., 1982, Un territoire sans nom : pour une approches des sociétés locales, Paris : Librairie des Méridiens, Coll. Sociologie au quotidien, $176 \mathrm{p}$.

Merlin P., 1997, « L'aménagement du territoire », in : Géographie humaine, Paris, PUF, pp. 381-422.

Mermet L., Treyer S., 2001, «Quelle unité territoriale pour la gestion durable de la ressource en eau? ", in : Les outils d'une bonne qualité de l'eau, Annales des Mines, Paris, Edition ESKA, Coll. Responsabilité et Environnement, pp. 67-79.

Meublat G., Le Lourd P., 2001, « Les Agences de Bassins : un modèle français de décentralisation pour les pays émergents. La rénovation des institutions de l'eau en Indonésie, au Brésil et au Mexique ", in : Les nouvelles politiques de l'eau. Enjeux urbains, ruraux, régionaux, Revue Tiers Monde, Meublat G. (Dir.), Paris, PUF, Tome XLII, n¹66, avril - juin, pp. 375-401.

Narcy J-B., 2004, Pour une gestion spatiale de l'eau. Comment sortir du tuyau ?, Bruxelles, P.I.E - Peter Lang, Ecoplis $\mathrm{n}^{\circ} 4,342 \mathrm{p}$.

Narcy J-B., 2003, «La politique de l'eau face à la gestion des espaces : les Agences de l'eau aux limites de la modernité ", in : Ambiances et espaces sonores, Espaces et sociétés, n¹15, pp. 179-196.

Narcy J-B.; Mermet L., 2003, « Nouvelles justifications pour une gestion spatiale de l'eau », in Natures, Sciences, Sociétés, Paris : Ed. Elsevier, Vol. 11, n², avril-juin, pp. 135-145.

Négrier E., 2001, « The changing role of french local government », communication au groupe CERAT, Les territoires dans l'action publique, séance sur les nouveaux territoires de l'intercommunalité, Grenoble, IEP, janvier, pp. 27-39.

Nicolazo J-L., 1997, Les agences de bassin, Paris, P. Johanet, 223 p.

Ozouf-Marignier M-V., 1989, La formation des Départements. La représentation du territoire français à la fin du $18^{\text {ème }}$ siècle, Paris, Editions de l'EHESS, 363 p.

Ozouf-Marignier M-V., 2002, « Bassins hydrographiques et divisions administratives en France ( XIX-XX ${ }^{\text {eme }}$ siècles) », in : Les nouvelles frontières de l'eau, Trames, CRDP Haute-Normandie, Académie de Rouen, $n^{\circ} 10$, pp. 63-76.

Petit D., 2002, « De l'espace protégé au territoire du développement, du réglementaire au contractuel. Expériences d'une réserve naturelle en Pyrénées-Orientales », in : Les arrière-pays aveyronnais et lozérien. L'innovation dans les IAA Européennes, Revue de l'Economie Méridionale, 4 - vol. $50, n^{\circ} 200$, pp. 385-406.

Picon B., 1978, L'espace et le temps en Camargue, essai d'écologie sociale, Le Paradou, Ed. Actes Sud, Coll. Espace-temps, 264 p. 
Poupeau F-M., 2004, Le service public à la française face aux pouvoirs locaux. Les métamorphoses de l'Etat jacobin, Paris, CNRS Editions, 336 p.

Pradeau C., 1999, « Réseaux hydrographiques et organisation de l'espace », L'Information Géographique, Paris, SEDES, n¹, vol. 63, pp. 3-9.

Puech D., Boisson J-M., 1995, « Eau-ressource et eau-milieu. Une interdépendance croissante impliquant une évolution des modalités de gestion ", in : Eau-ressource et eau-milieu vers une gestion durable, Les cahiers de l'Economie Méridionale, coll. Rapports d'étude, $\mathrm{n}^{\circ} 1$, pp. 5-47.

Raffestin C., 1986, « Ecogénése territoriale et territorialité », in Espaces, jeux et enjeux, Auriac F., B runet R., Paris, Fayard, pp. 175-185.

Raffestin C., 1980, Pour une géographie du pouvoir, Paris, Litec, Coll. Géographie économique et sociale, $249 \mathrm{p}$.

Reynoard E., 2001, « Aménagement du territoire et gestion de l'eau dans les stations touristiques alpine. Le cas de Crans-Montana-Aminona (Valais, Suisse) ", Revue de Géographie Alpine, $\mathrm{n}^{\circ} 3$, tome 89, pp. 7-19.

Ruf T., 2000, «Irrigation gravitaire et patrimoine commun, une approche des règles et usages dans les Pyrénées-Orientales », in L'irrigation gravitaire, Les journées techniques Agriculture Environnement, Chambres d'Agricultures PACA, Avignon, 14-15 septembre, pp. 185-198.

Thomé de Gamond A., 1871, «Plan d'ensemble pour la transformation de l'appareil hydraulique de la France », mémoire sur le régime général des eaux courantes, Paris, Dunod, librairie des corps des Ponts et Chaussées et des Mines, 90 p.

Turco A., 1997, « Aménagement et processus territoriaux : l'enjeu sémiologique », Espaces et Sociétés, n90/91, pp. 231-249.

Vanier M., 1997, « J'existe donc je découpe », in : Les découpages du territoire, INSEE METHODES, Lyon, Actes du colloque des dixièmes entretiens de Jacques Cartier, nº76-77-78, pp. 17-20.

Veltz P., 1996, Mondialisation, villes et territoire. L'économie d'archipel, Paris, PUF, 262 p.

Viard J., 1994, La société d'archipel ou les territoires du village global, Paris, La Tour d'Aigues, Editions de l'Aube, Coll. Monde en cours, 118 p.

Vieillard-Coffre S., 2001, « Gestion de l'eau et bassin versant », in Géopolitique de l'eau, Hérodote, Paris, La Découverte, $n^{\circ} 102$, pp. 139-156.

Wateau F., 2004, Les Conques d'Arbo, film ethnographique, 6'20, cellule audio-visuelle, CETSAH CNRS.

Wateau F., 2002a, Partager l'eau, irrigation et conflits au nord-ouest du Portugal, Paris, CNRS Editions, Editions de la maison des sciences de l'homme, $277 \mathrm{p}$.

Wateau F., 2002b, La pierre de partage de l'eau (co-réalisation d'Ana Margarida Campos), film ethnographique, 10 ', éditions du Laboratoire d'ethnologie et de sociologie comparative de Nanterre et du Musée national d'Ethnologie de Lisbonne, Lisboa.

Wateau F., 2001, « Objet et Ordre Social. D'une canne de roseau à mesurer l'eau aux principes de fonctionnement d'une société rurale », Terrain, 37, Paris : pp. 153-161. 


\section{NOTES}

1. Directive $2000 / 60 / C E$ du 23 octobre 2000 établissant une cadre pour une politique communautaire dans le domaine de l'eau, transposée en droit français par la loi $\mathrm{n}^{\circ}$ 2004-338 du 21 avril 2004.

2. Loi n64-1245 du 16 décembre 1964, (JO du 18 décembre 1964).

3. Loi n $92-3$ du 3 janvier 1992, (JO du 4 janvier 1992).

4. Directive cadre 2000/60 pour une politique communautaire dans le domaine de l'eau du Parlement et du Conseil européen du 23 octobre 2000 (JOCE nL327 du 22 décembre 2000).

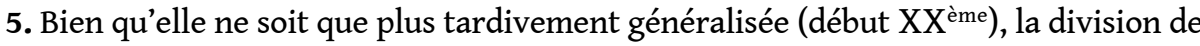
l'espace à partir de ses limites naturelles participe au découpage rationnel du territoire dont le but est la collecte d'informations relatives à l'eau. Jean-Paul Haghe (1998, p. 569) rappelle d'ailleurs que dans le cadre de la Seine, il s'agit véritablement d'une carte économique (approvisionnement en eau de Paris, prévision des crues) qui donna naissance au concept de région hydraulique (réunion du réseau physique et des activités humaines qui lui sont liées).

6. Relations entre l'Etat et le « local » pour l'appropriation des ressources, choix du territoire de gestion, statut juridique des cours d'eau, financement des infrastructures, incorporation des nouveaux usages (navigation, transport...).

7. Loi n¹919-10-16, du 16 octobre 1919 relative à l'utilisation de l'énergie hydraulique. (JO du 16 octobre 1919).

8. Les statuts sont approuvés le 11 octobre 1932 et la concession accordée le 15 juin 1934, (Haghe, 1998, pp. 549-559).

9. Compagnie d'Aménagement du Bas Rhône Languedoc, Société d'Aménagement du Canal de Provence, Compagnie d'Aménagement des Coteaux de Gascogne. Décret 55-876 du 30 juin 1955, relatif aux sociétés de développement régional (JO du 2 juillet 1955).

10. Loi n64-1245 du 16 décembre 1964, (JO du 18 décembre 1964).

11. gne et Rhône-

Méditerranée-Corse.

12. Loi no $92-3$ du 3 janvier 1992. (J.O. du 4 janvier 1992).

13. Modification de la Loi no $95-115$ du 4 février 1995, (J.O. du 13 juillet 1999).

14. Le CLE est la structure porteuse du SAGE, qui rassemble des élus, des usagers et des représentant de l'Etat. Ces acteurs locaux sont chargés de définir et de mettre en œuvre la politique de l'eau à l'échelle d'un bassin ou d'un sous-bassin.

15. La maîtrise du développement territorial passe ainsi non seulement par la capacité des acteurs à construire leur propre démarche de développement, mais également par leur capacité à construire des articulations et des agencements territoriaux combinant : les logiques sectorielles et globales, la dimension horizontale (territoire d'intervention élargi, gestion avec l'ensemble des acteurs locaux), la dimension essentielle, la dimension verticale (articulation avec les acteurs et les territoires d'intervention aux différentes échelles).

16. Citons notamment le manifeste de la décentralisation en Bretagne qui propose d'expérimenter la conduite de la politique de l'eau.

17. Les EPTB sont nés à l'initiative et dirigés le plus souvent par un ou plusieurs Conseils Généraux voire Régionaux. Ils sont le « bras armé » des élus locaux pour aménager et gérer les grands cours d'eau. 
18. Ces différentes catégories ne sont bien évidemment pas à considérer comme des entités homogènes.

\section{RÉSUMÉS}

La mise en place progressive en France d'une politique territoriale de l'eau, propulse la gestion de la ressource au cœur des enjeux liés à l'aménagement et au développement du territoire. Le vaste mouvement de recomposition organisationnelle et politique en cours favorise cette approche fonctionnaliste par bassin. Cependant, il contribue également à asseoir la légitimité d'autres constructions territoriales - dont les logiques relèvent davantage de l'opportunisme stratégique des collectivités territoriales que de la dimension géographique et environnementale de l'eau. A côté DU territoire de l'eau (ré ?) émergent ou se constituent DES territoires de l'eau.

The progressive implementation of territorial water policy in France, raises the stakes for water management in land planning and territorial development. The current vast movements in organisational and political reshuffling, support an operational approach by basin. However, it also contributes to establish the legitimacy of other territorial constructions - whose logic derives more from the strategic opportunism of local authorities than from the geographical and environmental dimensions of water. By side of "a" water territory, sits in fact, "many" water territories.

INDEX

Keywords : décentralisation, water policy, river basin, actors, stakes

Mots-clés : bassin versant, politique de l'eau, territorialisation, enjeux, acteurs, France

\section{AUTEUR}

\section{STÉPHANE GHIOTTI}

Stéphane Ghiotti, géographe, est chargé de recherches au cnrs. Il travaille sur la gestion territoriale de l'eau en France et dans le bassin méditerranéen (France, Liban, Maroc) au sein du laboratoire Mutation des Territoires en Europe (umr 5045), à Montpellier. Après une thèse soutenue en 2001 sur la place du bassin versant dans les dynamiques contemporaines du développement territorial, il a effectué entre 2002 et 2003 un postdoctorat au Département de Géographie de l'Université Saint Joseph de Beyrouth. Il poursuit actuellement ses recherches sur la réforme de la politique de l'eau au Liban. Laboratoire Mutations des Territoires en Europe (UMR - CNRS 5045), Bâtiment BRED, Université Paul Valéry - Montpellier III, Route de Mende ; 34199 Montpellier cedex 5 Tél. 0467142520 ; Fax. 0467142522 stephane.ghiotti@univ-montp3.fr 